\title{
On the Non-Real Roots of the Riemann Zeta Function $\varsigma(\mathrm{s})$
}

\section{Mustapha Azkour*}

Ministry of National Education, Vocational Training, Higher Education and Scientific Research, Kingdom of Morocco

\begin{abstract}
This paper aims to solve a very difficult problem closely related to analytic number theory and specifically to prime numbers. This problem is the Riemann hypothesis according to which the prime numbers follow a very regular distribution among natural numbers. The statement of the Riemann hypothesis is: "All the non-real roots of the complex function $\mathrm{s}(\mathrm{s})$ have real part equal exactly to $1 / 2$ ". In this paper, we give an original proof to this hypothesis proceeding by contradiction. This proof is exceptional and more special because it is based on the relevant property that when $\eta(s)=0$ where $\eta(s)$ is the Dirichlet eta function, the sum of the first $m-1$ terms of $\eta(s)$ converges to -0.5 multiplied by the $\mathrm{m}^{\text {th }}$ term with $\mathrm{m} \rightarrow \infty$ and it is also based on the condition that when we want to replace the complex variable $z$ by $z^{\prime}$ with $z^{\prime} \neq z$ in the expression of $\eta(z)$ which is defined on the half-plane $R(z)>0$ by $\eta(z)=\sum(-1)^{n-1} n^{-z}$, we have to keep the same infinite number of $n$-terms begining from $n=1$ to $n=\infty$ in order to converge exactly to the same function. After the exposure of the proof of the Riemann hypothesis, we give an original method combining two already existing for calculating the approximate value of the integral of any continuous real function fover a closed interval $\mathrm{I}=[\mathrm{a}, \mathrm{b}] \subset \mathbb{R}$ of course when the primitive of $f$ is not obvious by algebraic computation. This new numerical method is called " The Method of trapezes and half-ellipses THE" and its general approximation formula is defined by the following expression: $\int^{b} f(x) d x \approx T H E_{n}=(1-\gamma \pi / 4) T_{n}+(\gamma \pi / 4) M_{n}$ where $\mathrm{n}$ is the number of subintervals obtained by regular subdivision of the interval $[\mathrm{a}, \mathrm{b}]$ and $\gamma \in[\mathrm{O}, 4 / \pi]$ and $\mathrm{T}_{\mathrm{n}}$ is the approximate value of $\int^{b} f(x) d x$ using the method of trapezes and $\mathrm{M}_{\mathrm{n}}$ is the approximate value of $\int^{b} f(x) d x$ using the method of rectangles with midpoint and comparing the margins of error, we note that The Method of trapezes and half-ellipses THE is more accurate than the following three known methods: The method of rectangles on the left $R^{(1)}$, The method of rectangles on the right $R_{n}^{(v)}$ and The method of trapezes $T_{n}$.
\end{abstract}

Keywords: Riemann 's hypothesis; Riemann Zeta function; Nonreal roots of Riemann Zeta function; Dirichlet Eta function; Analytic number theory; Prime numbers; Numerical integration; The method of trapezes and half-ellipses THE

\section{Introduction}

The Riemann zeta function $\varsigma(\mathrm{s})$ plays a very important role in analytic number theory; its importance comes essentially from the very close connection it has with prime numbers; this connection that the great German mathematician Georg Friedrich Bernhard Riemann (1826-1866) had shown in his famous manuscript published in 1859(it is the same date when Charles Darwin (1809-1882) published his work "On the Origin of Species") when he gave an explicit formula linking the function counting the prime numbers with the roots of zeta function $\varsigma(s)$, namely the solutions $s \in \mathbb{C}$ of the equation $\varsigma(s)=0$, this explicit formula given by Riemann is $\pi(x)=L i(x)-\sum L i\left(x^{\rho}\right)-\log 2+\int d t / t\left(t^{2}-1\right) \log t$ where $\pi(\mathrm{x})$ is the function counting the prime numbers and $\mathrm{Li}$ is the Logarithmic integral defined by $L i(x)=\int d t / \log t$ and $\rho$ represents all the non-real roots of $\varsigma(s)$ and in his manuscript, Riemann claimed that it is very likely that all these non-real roots have real part equal exactly to $1 / 2$. The connection between the prime numbers and zeta function $\varsigma(\mathrm{s})$ which is defined on the half-plane $\mathfrak{R}(s) \geq 1$ by $\varsigma(s)=\sum n^{-s}$ was established before Riemann by the celebrated Euler (1707-1783) product: $\sum n^{-s}=\Pi\left(1-p^{-s}\right)^{-1}$ where the series $\sum n^{-s}$ is absolutely convergent for $\Re(s)>1$ and the product $\Pi\left(1-p^{-s}\right)^{-1}$ is being over the prime numbers $\mathrm{p} \in \mathrm{P}=\{2,3,5,7,11, \ldots\}$. So, in simple terms, the Riemann hypothesis says: "The non-real roots of $\varsigma(\mathrm{s})$ have all real part equal exactly to $1 / 2$ ". This has been checked for the first $10,000,000,000,000$ roots by experts and no counter-examples have been found. In practical terms, the Riemann hypothesis seems true, but theoretically, no proof to this moment has confirmed it. The validity of the Riemann hypothesis is equivalent to saying that the deviation of the number of the prime numbers from the mean $\operatorname{Li}(\mathrm{x})$ is $\pi(\mathrm{x})=\mathrm{Li}(\mathrm{x})+\mathrm{O}(\sqrt{\mathrm{x}} \log \mathrm{x})$. In this modest paper, we prove the absolute validity of the great Riemann hypothesis giving a very simple and rigorous proof which does not appeal to any complex theory and is very easy to understand.

\section{Some Proven Results about the Non-Real Roots of $\varsigma(s)$}

- The non-real roots of the Riemann zeta function $\varsigma(\mathrm{s})$ have all real part belonging to the critical strip ] O, 1 [and they are symmetric with respect to $\mathfrak{R}(s)=1 / 2$;

- In 1914, the British mathematician Godfrey Harold Hardy (1877-1947) proved that there are in finitely many roots of $\varsigma(\mathrm{s})$ on the critical line $\mathfrak{R}(\mathrm{s})=1 / 2$;

- The number of roots $s=\sigma+i$ of $\varsigma(s)$ in the critical strip ]O, $1[$ with $0<\mathrm{t} \leq \mathrm{T}$ is asymptotically equal to $\mathrm{N}(\mathrm{T})=(\mathrm{T} / 2 \pi) \log (\mathrm{T} / 2 \pi)$ $(\mathrm{T} / 2 \pi)+\mathrm{O}(\log \mathrm{T})$;

On the other hand, Hardy and Littlewood (1885-1977) had proved in the 1920 s a region without root of form $\mathfrak{R}(\mathrm{s})>1-\mathrm{k}(\log (\log \tau))(\log$

*Corresponding author: Mustapha Azkour, Ministry of National Education, Vocational Training, Higher Education and Scientific Research, Kingdom of Morocco, Tel: +21671766462; E-mail: mazkour@yahoo.com

Received May 14, 2018; Accepted June 22, 2018; Published June 30, 2018

Citation: Azkour M (2018) On the Non-Real Roots of the Riemann Zeta Function S(s). J Phys Math 9: 279. doi: 10.4172/2090-0902.1000279

Copyright: () 2018 Azkour M. This is an open-access article distributed under the terms of the Creative Commons Attribution License, which permits unrestricted use, distribution, and reproduction in any medium, provided the original author and source are credited. 
$\tau)-1$ where $\tau=|\mathrm{t}|+3, \mathrm{t}=\mathfrak{J}(\mathrm{s})$ and $\mathrm{k}$ is a positive constant;

And the Russian mathematician Ivan Vinogradov (1891-1983), in the thirties, had obtained a region without root of form $\mathfrak{R}(\mathrm{s})>1-\mathrm{k}$ $(\log \tau)^{-\gamma}$ where $\tau=|t|+3, t=\mathfrak{J}(s), 3 / 4<\gamma<1$ and $\mathrm{k}$ is a certain positive constant;

The largest known region that contains no roots of the function $\varsigma(\mathrm{s})$ is given asymptotically by $\mathfrak{R}(\mathrm{s})>1-\mathrm{k}(\log \tau)^{-2 / 3}(\log \log \tau)^{-1 / 3}$ where $\tau=|\mathrm{t}|$ $+3, t=\mathfrak{J}(\mathrm{s})$ and $\mathrm{k}$ is a certain positive constant;

In 1942, the Norwegian mathematician AtleSelberg (1917-2007) demonstrates that at least some fraction of roots of $\zeta(\mathrm{s})$ is on the critical line $\mathfrak{R}(\mathrm{s})=1 / 2$, but its fraction is less than $1 \%$;

In 1974, the American mathematician Norman Levinson (19121975) increases this fraction to $1 / 3$;

In 2004, the two mathematicians Xavier Gourdon and Patrick Demichel use the Odlyzko-Schonhage algorithm and find that the first $10,000,000,000,000$ roots of $\varsigma(\mathrm{s})$ are on the critical line $\mathfrak{R}(\mathrm{s})=1 / 2$;

In 2011, an other American mathematician John Brian Conrey (1955) proves that at least $41.05 \%$ of the non-real roots of $\varsigma(\mathrm{s})$ are aligned on the critical line $\mathfrak{R}(\mathrm{s})=1 / 2$ and are simple;

According to the Vinogradov-Korobov method, we have the following property: there are two constants $\mathrm{c}$ and $\mathrm{C}$ strictly positive such that for all $1 / 2 \leq \sigma \leq 1$ and $|t| \geq 2$, we have: $|\varsigma(\sigma+\mathrm{it})| \leq C|t| c(1-\sigma)^{3 / 2}(\log \mid t)^{2 / 3}$. In the current state of knowledge, according to the American mathematic cian Kevin Ford, we can take $\mathrm{c}=4.45$ and $\mathrm{C}=76.2$;

Littlewood also has proved the following theorem: "Either the function $\zeta(\mathrm{s})$ or the function $\varsigma^{\prime}(\mathrm{s})$ has an infinity of roots in the strip $1-\delta<\sigma<1, \sigma=\mathfrak{R}(\mathrm{s})$ and $\mathrm{o}$ is an arbitrarily small positive quantity";

In 1934, the Swiss mathematician Andreas Speiser (1885-1970) has proved the following theorem: ' $\forall \mathrm{s} \in \mathrm{C}$ with $0<\mathfrak{R}(\mathrm{s})<1 / 2: \varsigma^{\prime}(\mathrm{s}) \neq \mathrm{O}$ ";

The Turkish mathematician Gem Yalpn Yildirzm (1961) has proved that:

"The Riemann hypothesis implies that $\varsigma^{\prime \prime}(\mathrm{s})$ and $\varsigma^{\prime \prime}$ ( $(\mathrm{s})$ do not vanish in the strip $0<\Re(s)<1 / 2$ "; There are still more proven results about the non-real roots of ((s) apart from these results just mentioned, but all these results are not enough to say that the Riemann hypothesis is true. Fortunately, after several attempts we were able to solve this great problem and we believe that if there are many proofs to the Riemann hypothesis, our proof would probably be the simplest and the most beautiful.

\section{On the Non-Real Roots of $\varsigma(s)$}

\section{Key-question}

There is an important question we have to ask ourself, we wonder if any of the mathematicians who tried to prove the Riemann hypothesis and who failed if asked himself this question, it is the following keyquestion: "If $\mathrm{n}(\mathrm{s})=0$ and $\mathrm{n}(\mathrm{l}-\mathrm{s})=0$ and $0<\mathfrak{R}(\mathrm{s})<1$ and $\mathfrak{R}(\mathrm{s}) \neq 1 / 2$, then the limit $\lim _{z \rightarrow \delta} \eta(1-z) / \eta(z)$ has to be finite or infinite? it could be equal to 0 ? why not?". It is from this intelligent and relevant question and based on the explicit expression of $\eta(\mathrm{s})$ which is described as an infinite complex series for $\mathfrak{R}(\mathrm{s})>0$ and based on the special form of its general term $(-1)^{\mathrm{n}-1} \mathrm{n}^{-\mathrm{s}}$ and referring to universally known formulas and proven theorems that we started our reflection and our reasoning and carefully following the logical and rational implications we were able to find the right answer to the previous question and then to prove the absolute validity of the Riemann hypothesis and it should be noted here that the key result of the concise proof below lies in the expression of $\mathrm{n}(\mathrm{s})$.

\section{The proof of the Riemann hypothesis}

We know that the Riemann zeta function $\varsigma(\mathrm{s})$ is the analytic function of the complex variable $s$, defined on the half-plane $\mathfrak{R}(\mathrm{s})>1$ by $\varsigma(\mathrm{s})=\sum n^{-s}=\Pi\left(1-p^{-s}\right)^{-1} \quad$ [l] where the series $\sum n^{-s}$ is absolutely convergent for $\mathfrak{R}(s)>1$ and the product $\Pi\left(1-p^{-s}\right)^{-1}$ is being over the prime numbers $\mathrm{p} \in \mathrm{P}=\{2,3,5,7,11, \ldots\}$ and the function $\varsigma(\mathrm{s})$ is defined in the complex plane $\mathrm{Cl}\{\mathrm{l}\}$ by analytic continuation. As shown by Riemann, $\varsigma(\mathrm{s})$ can be continued analytically to $\mathrm{C} \backslash\{\mathrm{l}\}$ as a meromorphic function and has a first order pole at $s=1$ with residue 1 . On the other hand, we know that the Riemann zeta function $\varsigma(\mathrm{s})$ is defined for any complex number $s$ different from 1 and with real part strictly greater than 0 by $\varsigma(s)=\eta(s) /\left(1-2^{1-s}\right)$ [2] where $\eta$ is the Dirichl et eta function which is defined on the half-plane $\mathfrak{R}(\mathrm{s})>0$ by $\eta(\mathrm{s})=\sum(-1)^{n-1} n^{-s}$ [3]. Noticing that $\left(1-2^{1-s}\right) \neq \tilde{\infty}$ (remark: we denote $0=\{0, \mathrm{i} 0,0+\mathrm{i} 0\}$ and $\infty=\{\infty, \mathrm{i} \infty, \infty+\mathrm{i} \infty\}$ where $\left.\mathrm{i} \in \mathrm{C}, \mathrm{i}^{2}=-1\right)$ at least for $\mathfrak{R}(\mathrm{s})>0$, then we have to note the following result: if $\varsigma(\mathrm{s})=0$, then $\eta(\mathrm{s})=0$, we thus have the Riemann hypothesis is equivalent to the following statement: "The non-real roots of the Dirichet eta function $\eta$ (s) belonging to the critical strip ]0, 1 [have all real part equal exactly to $1 / 2$ ".

We also know that there is an important relationship between $\varsigma(\mathrm{s})$ and $\varsigma(1-\mathrm{s})$. This relationship is defined for any $\mathrm{s}$ in the complex plane $\mathbb{C}$ by the following Riemann functional equation: $\xi(s)=\xi(1-s)$ [4] where $\xi(s)=\pi^{-s / 2 T} \Gamma(s / 2) \varsigma(s)$, $\Gamma$ being the Euler gamma function which is defined for any complex number s such that $\mathfrak{R}(\mathrm{s})>0$ by: $\Gamma(\mathrm{s})=$ $\int_{0}^{\infty} x^{s-1} e^{-x} d x$ [5]. So, the two equations [2] and [4] imply: $\eta(1-\mathrm{s}) / \eta(\mathrm{s})=$ ${ }_{\Lambda}^{0}(s) / \Lambda(1-s)$ where: $\Lambda(s)=\pi^{-3 / 2} \Gamma(s / 2)\left(1-2^{s}\right)$. We also know that in 1896 , the two mathematicians Jacques Salomon Hadamard (1865-1963) and Charles-Jean de LaVallee Poussin (1866-1962) independently proved that no root of $\varsigma(\mathrm{s})$ could be on the line $\Re(s)=1$, and that all the nonreal roots have to be in the interior of the critical strip $0<\mathfrak{R}(\mathrm{s})<1$, and it is known that this demonstration was a key result in the first complete proof of the prime number theorem $\left(P N T: \lim _{x \rightarrow \infty} \eta(1-z) / \eta(z)=0, \tilde{\infty}\left(^{*}\right)\right)$. Now, let 's assume that there is a complex number s such that $0<\mathfrak{R}$ (s)<1 verifying $\lim _{x \rightarrow s} \Lambda(z) / \Lambda(1-z) \neq 0, \tilde{\infty}$. According to this assumption, we have to have: $\lim _{x \rightarrow s} \Lambda(z) / \Lambda(1-z)=0, \infty$ So, we have if $\mathfrak{R}$ (s), $\mathfrak{R}$ (s)) $\neq(0,1)$ and knowing that $\Gamma(s)$ does not vanish for any $s$ in $\mathbb{C}$ and has an infinity of simple poles with residue $(-1)^{\mathrm{k}} / \mathrm{k}$ ! at $s=-\mathrm{k}$ where $\mathrm{k}=0,1$, $2,3,4,5, \ldots$ etc. then $\lim _{x \rightarrow s} \Lambda(z) / \Lambda(1-z)=0 \Rightarrow s=3,5,7,9,11, \ldots$ etc. and $\lim _{x \rightarrow s} \Lambda(z) / \Lambda(1-z)=\tilde{\infty} \Rightarrow s=-2,-4,-6,-8,-10, \ldots$ etc. then we have for $0<$ $\mathfrak{R}(\mathrm{s})<1: \lim \Lambda(z) / \Lambda(1-z) \neq 0, \tilde{\infty}$, that is to say the assumption $\left(^{*}\right)$ is false. Thus, for every complex number s such that $0<\mathfrak{R}(\mathrm{s})<1$, we have to be sure that $\lim _{x \rightarrow \infty} \eta(1-z) / \eta(z)=0, \tilde{\infty}$. Therefore, we have to mention the following property: if $\eta(\mathrm{s})=0$ and $0<\mathfrak{R}(\mathrm{s})<1$, then $\eta(1-\mathrm{s})=0$.

Important remark: This proof is mainly based on the following known data:

- If $\varsigma(s)=0$ and $s \neq-2,-4,-6, \ldots$ etc. then $0<\mathfrak{R}(s)<1$;

$\varsigma(\mathrm{s})=\eta(\mathrm{s}) /\left(1-2^{1-\mathrm{s}}\right), \eta$ is defined for $\mathfrak{R}(\mathrm{s})>0$ by $\eta(\mathrm{s})=\sum(-1)^{n-1} n^{-s}$;

For any s in $C: \xi(s)=\xi(1-s)$ where $\xi(s)=\pi^{-s / 2} \Gamma(s / 2) \varsigma(s)$;

$\forall|x| \leq 1: \lim _{t \rightarrow x} \sum_{n \geq 0}(-1)^{n} t^{n}=\lim _{t \rightarrow x} \frac{1}{1+t}$ 
In general $\lim _{x \rightarrow x_{0}} \frac{f(x)}{g(x)} \neq \frac{\lim _{x \rightarrow x_{0}} f(x)}{\lim _{x \rightarrow x_{0}} g(x)}$,

If $\eta_{m}(z)=\sum_{n=1}^{m}(-1)^{n-1} n^{-z}$ then $\left\{\eta(s)=\lim _{z \rightarrow s} \eta_{m}(z)\right.$ and $\left.\eta\left(s^{\prime}\right)=\lim _{m^{\prime} \rightarrow s^{*}}, \eta_{\mathrm{m}^{*}}\left(z^{\prime}\right)\right\} \Rightarrow m^{*}=m$ , applying this condition we keep the same infinite number of $\eta$ - terms in order to converge exactly to the same function in numerator and denominator,

To prove the Riemann hypothesis, it seemed to us that it is more convincing to prove the conjecture:

If $\eta(\mathrm{s})=0$ and $0<\mathfrak{R}(\mathrm{s})<1$, then: $\lim _{z \rightarrow s} \frac{\eta(1-z)}{\eta(z)}=0, \tilde{\infty}$ or $\mathfrak{R}(\mathrm{s})=\frac{1}{2}[C]$

In the conjecture above, we denote: $0=\{0, \mathrm{i} 0,0+\mathrm{i} 0\}$ and $\tilde{\infty}=\{\infty, \mathrm{i} \infty$, $\infty+i \infty\}$ where $i \in \mathbb{C}$,

The proof of the conjecture [C] [algebraic proof]: We have previously shown that for every complex number s such that $0<\Re$ (s) $<1$, we have $\lim _{z \rightarrow s} \eta(1-z) / \eta(z) \neq 0, \tilde{\infty}$ and we have deduced according to this result that if we have $\eta(s)=0$ and $0<\mathfrak{R}(\mathrm{s})<1$, then we also must have $\eta(1-\mathrm{s})=0$, and we know that $\eta$-function is defined on the halfplane $\eta(\mathrm{s})>0$ by $\eta(\mathrm{s})=\sum \alpha_{n}(s)$ where $\alpha_{\mathrm{n}}(\mathrm{s})=(-1)^{\mathrm{n}-1} \mathrm{n}^{-\mathrm{s}}$.

To prove the conjecture $[\mathrm{C}]$, we have to prove the following proposition:

If $\quad \eta \quad(\mathrm{s})=0, \quad$ and $\quad \eta \quad(1-\mathrm{s})=0 \quad$ and $\quad 0<\mathfrak{R}(\mathrm{s})<1, \quad$ then $\lim _{z \rightarrow s} \frac{\eta(1-z)}{\eta(z)}=\lim _{\substack{z \rightarrow s \\ m \rightarrow \infty}} \frac{\alpha_{m}(1-z)}{\alpha_{m}(z)}$

So, let's prove the proposition $[\mathrm{P}]$,

Proof: We have:

$$
\eta(z)=\sum_{n=1}^{m-1} \alpha_{n}(z)+\sum_{n \geq m} \alpha_{n}(z), m \geq 2
$$

We denote:

$$
\eta_{m}(z)=\sum_{n=1}^{m-1} \alpha_{n}(z) \text { and } \eta_{\geq m}(z)=\sum_{n \geq m} \alpha_{n}(z)
$$

So, we have

$$
\lim _{z \rightarrow s} \frac{\eta(1-z)}{\eta(z)}=\lim _{\substack{z \rightarrow s \\ m \rightarrow \infty}} \frac{\eta_{m}(1-z)+\eta_{\geq m}(1-z)}{\eta_{m}(z)+\eta_{\geq m}(z)}
$$

and for any $s$ in the complex plane $\mathbb{C}$ with $\mathfrak{R}(\mathrm{s})>0$, we have: $\lim _{z \rightarrow s} \eta_{\geq m}(z)=0$ that is to say if then

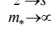

$$
\begin{aligned}
& \lim _{\substack{z^{\prime} \rightarrow s \\
m_{*} \rightarrow \infty}} \eta_{\geq m}(z) \neq 0 \text { we have: } \\
& \lim _{\substack{z \rightarrow s \\
m \rightarrow \infty}} q_{m}(z)=0 \text { where } q_{m}(z)=\frac{\eta_{\geq m}(z)}{\eta_{m}(z)}
\end{aligned}
$$

but if $\lim _{\substack{z \rightarrow s \\ m \rightarrow \infty}} \eta_{m}(z)=0$, it will still remain $\lim _{\substack{z \rightarrow s \\ m \rightarrow \infty}} q_{m}(z)=0$ ? in the following, we are going to prove $\lim _{\substack{z \rightarrow s \\ m \rightarrow \infty}} \eta_{m}(z)=0$ then $\lim _{\substack{z \rightarrow s \\ m \rightarrow \infty}} q_{m}(z) \neq 0$, so in general we can simply write:

$$
\lim _{z \rightarrow s} \frac{\eta(1-z)}{\eta(z)}=\lim _{\substack{z \rightarrow s \\ m \rightarrow \infty}} \frac{\eta_{m}(1-z)}{\eta_{m}(z)}
$$

If $\mathrm{m}^{*}=\mathrm{m}\left(\mathrm{m}_{*=} \mathrm{m}\right.$ is a necessary condition in the following calculation of limits), then we have:

$$
\begin{aligned}
& \eta(s)=\lim _{\substack{z \rightarrow s \\
m \rightarrow \infty}}\left(\eta_{m}(z)+\eta_{\geq m}(z)\right)=\lim _{\substack{z \rightarrow s \\
m \rightarrow \infty}} \eta_{m}(z)+\lim _{\substack{z \rightarrow s \\
m_{*} \rightarrow \infty}} \eta_{m \geq m}(z) \\
& \text { And } \forall_{\mathrm{n}} \geq 1 \text { and } \forall_{\mathrm{j}} \geq 0 \text {, we have: } \alpha_{n+j}(z)=(1)^{j}\left(\frac{n}{n+j}\right)^{z} \alpha_{n}(z),
\end{aligned}
$$

So, for $\mathrm{m}_{*}=\mathrm{m}$, we have

$$
\begin{aligned}
& \lim _{\substack{z \rightarrow s \\
m_{*} \rightarrow \infty}} \sum_{\substack{ \\
m_{*}}} \alpha_{n}(z)=\lim _{\substack{z \rightarrow s \\
m \rightarrow \infty}}\left(\alpha_{m}(z)+\sum_{j \geq 1} \alpha_{m+j}(z)\right) \\
& =\lim _{\substack{z \rightarrow s \\
m_{*} \rightarrow \infty}}\left(\alpha_{m_{*}}(z)+\sum_{j \geq 1}(-1)^{j}\left(\frac{m_{*}}{m_{*}+j}\right)^{z} \alpha_{m^{*}}(z)\right) \\
& =\lim _{\substack{z \rightarrow s \\
m_{*} \rightarrow \infty}}\left(\alpha_{m}(z)+\left(1+\sum_{j \geq 1}(-1)^{j}\left(\frac{m}{m+j}\right)^{z}\right)\right)
\end{aligned}
$$
write:

It's trivial that if $\lim _{x \rightarrow x_{0}} f(x)$ exists and $\lim _{x \rightarrow x_{0}} g(x)$ exists, then we can

$$
\lim _{x \rightarrow x_{0}} f(x) g(x)=\lim _{x \rightarrow x_{0}} f(x) \lim _{x \rightarrow x_{0}} g(x)
$$

We have $\lim _{\substack{z \rightarrow s \\ m \rightarrow \infty}} \alpha_{m}(z)$ exists for $\mathfrak{R}(\mathrm{s})>0$ and is equal to 0 , but we have to prove that $\mathrm{L}$ also exists,

where:

$$
\lim _{\substack{z \rightarrow s \\ m \rightarrow \infty}}\left(1+\sum_{j \geq 1}(-1)^{j}\left(\frac{m}{m+j}\right)^{z}\right)
$$

We have

$$
\underset{\substack{z \rightarrow s \\ m \rightarrow \infty}}{L=\lim _{m \rightarrow 1}}\left(1+\sum_{j \geq 1}(-1)^{j}\left(\frac{m}{m+j}\right)^{z}\right)=\lim _{\substack{z \rightarrow s \\ m \rightarrow \infty}}\left(1+\sum_{j \geq 1}(-1)^{j}\left(\left(\frac{m}{m+j}\right)^{z / j}\right)^{j}\right)
$$

Denoting $\left(\frac{m}{m+j}\right)^{z / j}$ by $\mathrm{x}_{\mathrm{j}}(\mathrm{z}, \mathrm{m})$, we note that $\forall \mathrm{j}, \mathrm{m} \geq 1$

$\left|x_{j}(z, m)\right| \leq 1$

That means $\forall \mathrm{j}, \mathrm{m} \geq 1: \exists \in \mathrm{j}(\mathrm{z}, \mathrm{m})>0$ verifying $\left|x_{j}(z, m)\right| \leq 1-\in j(z, m)$ and when $\mathrm{m} \rightarrow \infty: \mathrm{ej}(\mathrm{z}, \mathrm{m}) \rightarrow 0$,

That is to say $\forall j, j^{\prime} \geq 1$ with $j \neq j^{\prime}$ when $\mathrm{z}, \mathrm{m} \rightarrow \mathrm{s}, \infty$

$x_{j}(z, m)=x_{j^{\prime}}(z, m)=1^{-}+i 0$

And we know that $\forall|x|<1: \lim _{t \rightarrow x} \sum_{n \geq 0}(-1)^{n} t^{n}=\lim _{t \rightarrow x} \frac{1}{1+t}$

We thus get,

$$
\begin{aligned}
& \underset{\substack{z \rightarrow s \\
m \rightarrow \infty}}{L}=\lim _{\substack{m \\
\text { M }}}\left(1+\sum_{j \geq 1}(-1)^{j} x_{j}(z, m)^{j}\right)=1+\lim _{\substack{z \rightarrow s \\
m \rightarrow \infty}} \sum_{j \geq 1}(-1)^{j} x_{j}(z, m)^{j} \\
& =1+\lim _{\substack{z \rightarrow s \\
m \rightarrow \infty}} \frac{1}{1+x_{j}(z, m)}-1=1+\frac{1}{1+1}-1=\frac{1}{2}=0.5
\end{aligned}
$$

So, for $\mathrm{m}_{*}=\mathrm{m}$ (we must not forg et that $\mathrm{m}_{*}=\mathrm{m}$ is a necessary condition in calculation of limits):

$$
L=\lim _{\substack{z \rightarrow s \\ m_{*} \rightarrow \infty}} \eta_{\geq m *}(z)=0.5 \lim _{\substack{z \rightarrow s \\ m \rightarrow \infty}} \sum_{j \geq 1}(-1)^{j} x_{j}(z, m)^{j}
$$


That is to say if $z, m \rightarrow s, \infty$ with $\Re(s)>0$, then:

$\eta(z)=\eta_{m}(z)+0.5 \alpha_{m}(z)$

So, if $\mathrm{n}(\mathrm{z}) \rightarrow 0$ when $\mathrm{z}, \mathrm{m} \rightarrow \mathrm{s}, \infty$, then we have:

$\eta_{m}(z) \rightarrow-0.5 \alpha_{m}(z)$

We have to note that if $\lim _{\substack{z \rightarrow s \\ m \rightarrow \infty}} \eta_{m}(1-z)=0$ and $\lim _{\substack{z \rightarrow s \\ m \rightarrow \infty}} \eta_{m}(z)=0$ then, $\lim _{z \rightarrow s} \frac{\eta(1-z)}{\eta(z)} \neq \frac{\lim _{\substack{z \rightarrow s \\ m \rightarrow \infty}} \eta_{m}(1-z)}{\lim _{\substack{z \rightarrow s \\ m \rightarrow \infty}} \eta_{m}(z)}=\frac{0}{0}$

So, according to $\left({ }^{*}\right)$, it follows that if $\mathrm{n}(\mathrm{s})=0$ and $\mathrm{n}(\mathrm{l}-\mathrm{s})=0$, then we must have:

$$
\lim _{z \rightarrow s} \frac{\eta(1-z)}{\eta(z)}=\lim _{\substack{z \rightarrow s \\ m \rightarrow \infty}} \frac{\eta_{m}(1-z)}{\eta_{m}(z)}=\lim _{\substack{z \rightarrow s \\ m \rightarrow \infty}} \frac{-0.5 \alpha_{m}(1-z)}{\alpha(1-z)}=\lim _{\substack{z \rightarrow s \\ m \rightarrow \infty}} \frac{\alpha_{m}(1-z)}{\alpha_{m}(z)} \equiv[\mathbf{P}]
$$

So, the proposition $[\mathbf{P}]$ implies the following result:

$$
\begin{aligned}
& \text { If } \quad \mathrm{n}(\mathrm{s})=0 \quad \text { and } \quad 0<\mathfrak{R}(\mathrm{s})<1 \text { and } \quad \mathfrak{R}(s) \neq \frac{1}{2}, \quad \text { then } \\
& \lim _{z \rightarrow s} \frac{\eta(1-z)}{\eta(z)}=\lim _{\substack{z \rightarrow s \\
m \rightarrow \infty}} \frac{\alpha_{m}(1-z)}{\alpha_{m}(z)}=0, \tilde{\infty}
\end{aligned}
$$

Thus it has been shown that:

If $\mathrm{n}(\mathrm{s})=0$ and $0<\mathfrak{R}(\mathrm{s})<1$ [, then $\lim _{z \rightarrow s} \frac{\eta(1-z)}{\eta(z)}=0, \tilde{\infty}$ or $\mathfrak{R}(s)=\frac{1}{2} \equiv[\mathrm{C}]$

So, if $\mathrm{n}(\mathrm{s})=0$ and $\mathfrak{R}(\mathrm{s}) \in] 0,1 / 2[\mathrm{U}] 1 / 2,1\left[\right.$, then $\lim _{z \rightarrow s} \frac{\eta(1-z)}{\eta(z)}=0, \tilde{\infty}$, that is equivalent to say that $\lim _{z \rightarrow s} \frac{\Lambda(1-z)}{\Lambda(z)}=0, \tilde{\infty}$ [we remind here that $\mathrm{A}(\mathrm{z})=\pi^{-\mathrm{z} / 2 \mathrm{r}}(\mathrm{z} / 2)\left(1-2^{z}\right) \mathrm{J}$. However, for any complex $\mathrm{s}$ with number $\mathrm{s}$ with $0<\mathfrak{R}(\mathrm{s})<1$, we have shown that $\lim _{z \rightarrow s} \frac{\Lambda(1-z)}{\Lambda(z)} \neq 0, \tilde{\infty}$. Therefore,
we deduce that:

if $\mathrm{n}(\mathrm{s})=0$ and $0<\mathfrak{R}(\mathrm{s})<1$, then $\mathfrak{R}(\mathrm{s})=1 / 2$, and according to $[\mathbf{P}]$, we also note that:

if $\mathrm{y}(\mathrm{s})=0$ and $0<\mathfrak{R}(\mathrm{s})<1$, then $\mathfrak{J}(\mathrm{s})=\pi \mathrm{x} / \log \mathrm{y}$ where $\mathrm{x} \in \mathrm{Z}^{*}$ and $\mathrm{y}$ $\in] 0,1[U] 1+\infty[$.

The proposition [P] can be generalized by the following statement: $\forall s, s^{\prime} \in \mathbb{C}$ with $\Re(s), \Re\left(s^{\prime}\right) \geq 0$, if $\eta(s)=0$ and $\eta\left(s^{\prime}\right)=0$, then

$$
\lim _{\substack{z \rightarrow s \\ z^{\prime} \rightarrow s^{\prime}}} \frac{\eta(z)}{\eta\left(z^{\prime}\right)}=\lim _{\substack{z \rightarrow s \\ z^{\prime} \rightarrow s^{\prime} \\ m \rightarrow \infty}} \frac{\alpha_{m}(z)}{\alpha_{m}\left(z^{\prime}\right)}
$$

- For example, for $\mathrm{t}$ being the imaginary part of a non-real root of $\varsigma(\mathrm{s})$ and belonging to the critical line $\mathfrak{R}(\mathrm{s})=\sigma=1 / 2$ and $\mathrm{kE} \mathbb{Z}^{*}$, we have:

$$
\left.\lim _{\substack{z \rightarrow 1 / 2+i t \\ z^{\prime} \rightarrow i 2 k \pi / \log 2}} \frac{\eta(z)}{\eta\left(z^{\prime}\right)}=\lim _{\substack{z \rightarrow 1 / 2+i t \\ z^{\prime} \rightarrow i 2 k \pi / \log 2 \\ m \rightarrow \infty}} \frac{\alpha_{m}(z)}{\alpha_{m}\left(z^{\prime}\right)}=\lim _{m \rightarrow \infty} m^{1 / 2+i((2 k \pi / \log 2)-t)}=\tilde{\infty}{ }_{(\star *}\right)
$$

If we look for more precision and rigor, we have to take into consideration in our calculation of limits the following obvious condition (note: this condition is respected in the previous calculation of limits) [6]:

$$
\text { *If } \eta_{m}(z)=\sum_{n=1}^{m}(-1)^{n-1} n^{-z}, \text { then }\left\{\eta(s)=\lim _{\substack{z \rightarrow s \\ m \rightarrow \infty}} \eta_{m}(z) \text { and } \eta\left(s^{\prime}\right)=\lim _{\substack{z \rightarrow s^{\prime} \\ m_{*} \rightarrow \infty}} \eta_{m_{*}}\left(z^{\prime}\right)\right\}
$$

$$
\Rightarrow \lim _{\substack{z \rightarrow s^{\prime} \\ z^{\prime} \rightarrow s^{\prime}}} \frac{\eta(z)}{\eta\left(z^{\prime}\right)}=\lim _{\substack{z \rightarrow s^{\prime} \\ z^{\prime} \rightarrow s^{\prime} \\ m \rightarrow \infty}} \frac{\eta_{m}(z)}{\eta_{m}\left(z^{\prime}\right)} *
$$

By this condition, we want to say that if someone wants to replace $z$ by $z^{\prime}$ with $z^{\prime} \neq z$ in the expression of $\mathrm{n}$, he has to keep the same number of $n$-terms beginning from $n=1$ to $n=m=\infty$ to keep exactly the same function and to change only the complex variable $\mathrm{z}$ in the expression of $\mathrm{n}$ and this condition is valid in the general case.

In short, the most interesting result proved in this paper is the following theorem:

If $\varsigma(\mathrm{s})=0$ and $\mathfrak{R}(\mathrm{s})>0$, then $\mathfrak{R}(\mathrm{s})=1 / 2$ and $\mathfrak{J}(\mathrm{s})=\pi \mathrm{x} / \log \mathrm{y}$ where $\mathrm{x} \in$ $\mathbb{Z}^{*}$ and $\left.\mathrm{y} \in\right] 0,1[\mathrm{U}] 1,+\infty[$.

Numerical verification: We have made the numerical verification for $\left({ }^{*}\right)$ and $\left({ }^{* *}\right)$ using the first 5 roots of the Riemann zeta function $\varsigma(\mathrm{s})$ whose real parts are equal to $1 / 2$ and the imaginary parts are positive and we should note that this numerical verification was done using Microsoft Office Excel 2007,

The first 5 roots of the Riemann zeta function $\varsigma(\mathrm{s})$ are:

$\mathrm{S} 1=1 / 2+\mathrm{i} 14,134725141 \ldots$

$\mathrm{S} 2=1 / 2+\mathrm{i} 21,022039638 \ldots$

$\mathrm{S} 3=1 / 2+\mathrm{i} 25,010857580 \ldots$

$\mathrm{S} 4=1 / 2+\mathrm{i} 30,424876125 \ldots$

$\mathrm{S} 5=1 / 2+\mathrm{i} 32,935061587 \ldots$

and using the first root of the Dirichlet eta function $\mathrm{n}(\mathrm{s})$ whose real part is equal to 1 and the imaginary part is equal to $2 \pi / \log 2$ in order to show that $\lim _{\substack{z \rightarrow s \\ z^{\prime} \rightarrow s^{\prime}}} \frac{\eta_{m}(z)}{\eta_{m}(z)}=\tilde{\infty}$ where sj represents all the nonreal roots of ((s) aligned on the critical line at $\mathfrak{R}(\mathrm{s})=\sigma=1 / 2$ and $s_{1}^{\prime} i s: s^{\prime}=1+i 2 \pi / \log 2$,

In this verification we have taken: $\eta_{m}(s)=\sum_{n=1}^{m-1} \alpha_{n}(s)$ with $a_{\mathrm{n}}(\mathrm{s})=(-1)^{\mathrm{n}-1} \mathrm{n}^{-\mathrm{s}}=\alpha \mathrm{n}(\mathrm{s})+\mathrm{ib} \mathrm{n}_{\mathrm{n}}(\mathrm{s})$

See the calculation tables in the next pages: from [09/26] to [21/26] and tables [1-13]. The paces of the two quotients $\left|\eta_{m}\left(s_{1}\right) / \eta_{m}\left(s_{1}^{\prime}\right)\right| \xi\left|\alpha_{m}\left(s_{1}\right) / \alpha_{m}\left(s_{1}^{\prime}\right)\right|$ for $\mathrm{m}$ ranging from 10 to 1000000 on the page [22/26] (Figure 1 and Tables 1-13).

\section{Conclusion}

Based on the following condition:

$$
\begin{aligned}
& \text { *If } \eta_{m}(z)=\sum_{n=1}^{m}(-1)^{n-1} n^{-z}, \text { then }\left\{\eta(s)=\lim _{\substack{z \rightarrow s \\
m \rightarrow \infty}} \eta_{m}(z) \text { and } \eta\left(s^{\prime}\right)=\lim _{\substack{z \rightarrow s^{\prime} \\
m \rightarrow \infty}} \eta_{m}\left(z^{\prime}\right)\right\} \Rightarrow m_{*}=m \\
& \Rightarrow \lim _{\substack{z \rightarrow s^{\prime} \\
z^{\prime} \rightarrow s^{\prime}}} \frac{\eta(z)}{\eta\left(z^{\prime}\right)}=\lim _{\substack{z \rightarrow s^{\prime} \\
z^{\prime} \rightarrow s^{\prime} \\
m \rightarrow \infty}} \frac{\eta_{m}(z)}{\eta_{m}\left(z^{\prime}\right)} *
\end{aligned}
$$

and on the special property that when $\mathrm{n}(\mathrm{s})=0$ for $\mathfrak{R}(\mathrm{s})>0$ and $\mathrm{z}, \mathrm{m} \rightarrow \mathrm{s}, \infty$ :

$$
\eta_{m}(z) \rightarrow-0.5 \alpha_{m}(z) \text { where }_{m}(z)=\sum_{n=1}^{m-1} \alpha_{n}(z) \text { and } \alpha_{n}=(-1)^{n-1} n^{-z}
$$

it has been shown that if $\mathrm{n}(\mathrm{s})=0$ and $\mathrm{n}(\mathrm{l}-\mathrm{s})=0$ with $0<\mathfrak{R}(\mathrm{s})<1$, then we get: 


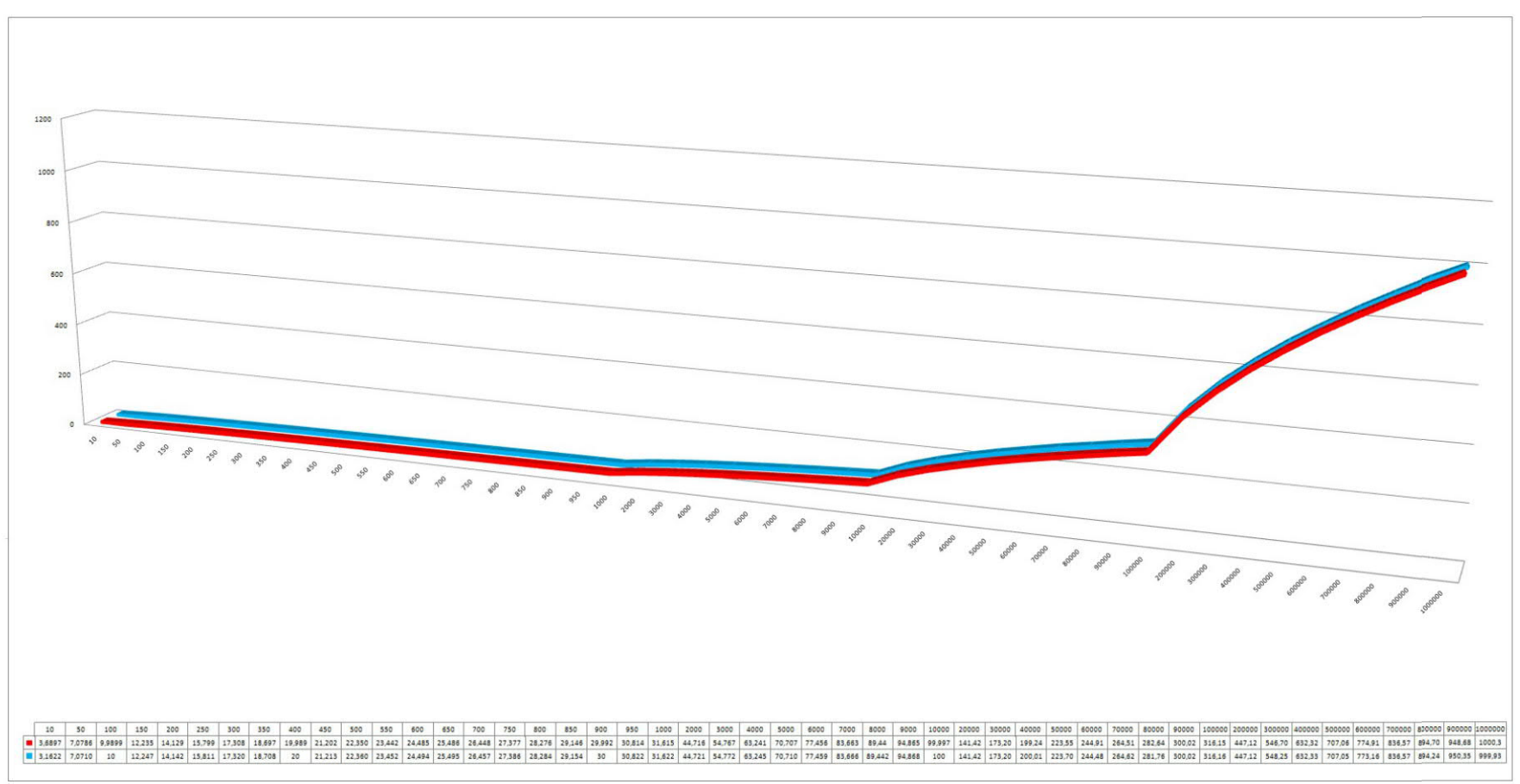

Figure 1: The paces of the two quotients $11^{\prime} / \mathrm{rn}(\mathrm{s} 1) / 77 \mathrm{rn}(\mathrm{sDI} 8 / \mathrm{arn}(\mathrm{s})) / /$ for $\mathrm{m}$ ranging from 10 to 1000000 .

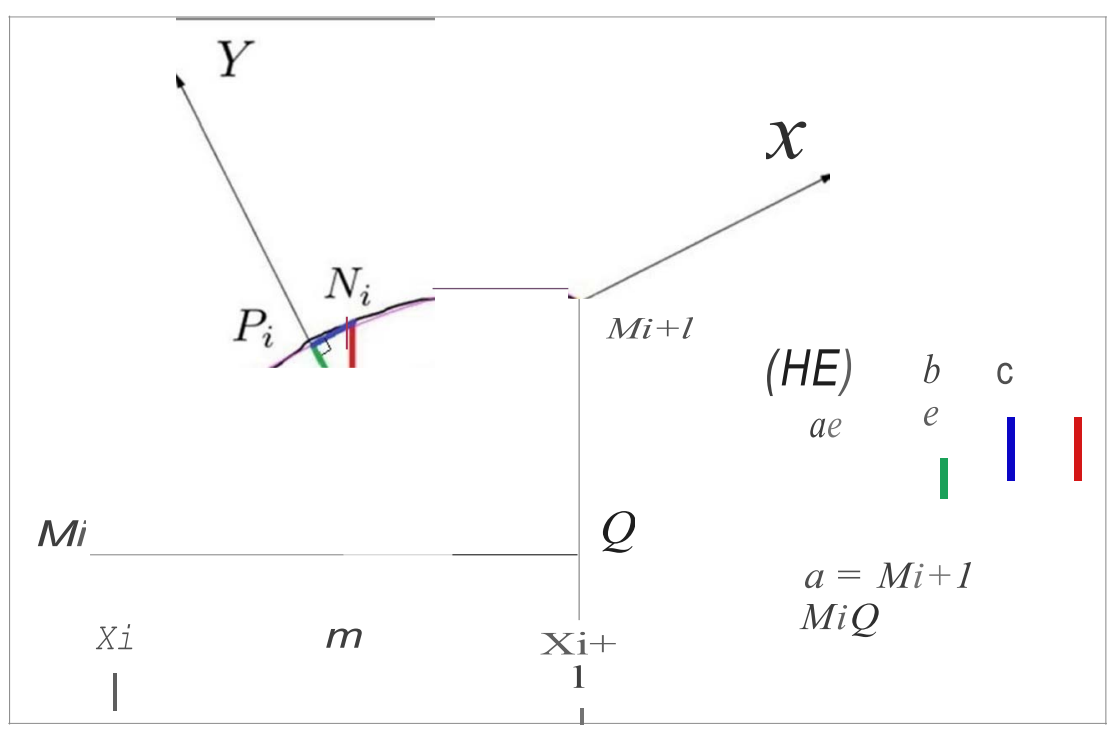

Figure 2: Chart explaining the existence and purpose of method of Trapezes and Half-ellipses T.H.E.

$$
\lim _{z \rightarrow s} \frac{\eta(1-z)}{\eta(z)}=\lim _{\substack{z \rightarrow s \\ m \rightarrow \infty}} \frac{\eta_{m}(1-z)}{\eta_{m}(z)}=\lim _{\substack{z \rightarrow s \\ m \rightarrow \infty}} \frac{-0.5 \alpha_{m}(1-z)}{-0.5 \alpha_{m}(z)}=\lim _{\substack{z \rightarrow s \\ m \rightarrow \infty}} \frac{\alpha_{m}(1-z)}{\alpha_{m}(z)}
$$

and refering to this result, it has been deduced that:

$$
\text { If } \eta(s)=0 \text { and } 0 \leq \mathfrak{R}(s) \leq 1 \text {, then } \lim _{z \rightarrow s} \frac{\eta(1-z)}{\eta(z)}=0 . \tilde{\infty} \text { or } \Re(s)=\frac{1}{2}
$$

and it is also proved that if $\mathrm{n}(\mathrm{s})=0$ and $0<\mathfrak{R}(\mathrm{s})<1$, then $\mathfrak{J}(\mathrm{s})=\pi \mathrm{x} / \log \mathrm{y}$ where $\mathrm{x} \in \mathbb{Z}^{*}$ and

$$
\mathrm{y} \in] 0,1[\mathrm{U}] 1,+\infty[\text {. }
$$

\section{The Method of Trapezes and Half-Ellipses T.H.E}

\section{Purpose}

This method is original and its main purpose is the approximate calculation of the integral of any continuous real function $\mathrm{f}$ on a closed interval $[a, b]$ with $a<b$ replacing each arc of $f$-curve $\left(M_{i} M_{i+1}\right)$ by an elliptical half-arc (H E) and we remind that this method can be used 


\begin{tabular}{|c|c|c|c|}
\hline$m$ & ${ }^{R}\left(\mathbf{n}_{\mathrm{m}}\left(\mathbf{s}_{1}\right)\right)$ & $a_{m}\left(s_{1}\right)$ & $\frac{\Re\left(\eta_{m}\left(s_{1}\right)\right)}{a m\left(s_{1}\right)}$ \\
\hline 10 & 0,192867143 & $-0,134784134$ & $-1,43,09,33,577$ \\
\hline 50 & 0,01263537 & $-0,044150483$ & $-0,286188715$ \\
\hline 100 & $-0,029184638$ & 0,063666475 & $-0,458398835$ \\
\hline 150 & $-0,003723617$ & 0,011241463 & $-0,331239537$ \\
\hline 200 & 0,030322087 & $-0,06178251$ & $-0,490787555$ \\
\hline 250 & $-0,027422519$ & 0,055639564 & $-0,492860063$ \\
\hline 300 & 0,013530651 & $-0,028224466$ & $-0,479394402$ \\
\hline 350 & 0,01216527 & $-0,023342749$ & $-0,521158498$ \\
\hline 400 & $-0,024727443$ & 0,049543065 & $-0,499110077$ \\
\hline 450 & $-0,001342723$ & 0,001944584 & $-0,690493699$ \\
\hline 500 & 0,022164627 & $-0,044384495$ & $-0,499377699$ \\
\hline 550 & 0,007500051 & $-0,014478126$ & $-0,518026366$ \\
\hline 600 & $-0,015631457$ & 0,031554882 & $-0,495373648$ \\
\hline 650 & $-0,017808192$ & 0,035419543 & $-0,502778706$ \\
\hline 700 & $-0,001687111$ & 0,002992738 & $-0,563734948$ \\
\hline 750 & 0,0141525 & $-0,028510481$ & $-0,496396395$ \\
\hline 800 & 0,017224078 & $-0,034363969$ & $-0,501224931$ \\
\hline 850 & 0,007995398 & $-0,015732746$ & $-0,508201048$ \\
\hline 900 & $-0,005300603$ & 0,010845709 & $-0,488728123$ \\
\hline 950 & $-0,014373582$ & 0,028849992 & $-0,498217885$ \\
\hline 1000 & $-0,015351898$ & 0,030640875 & $-0,501026749$ \\
\hline 2000 & 0,009107231 & $-0,018166118$ & $-0,501330609$ \\
\hline 3000 & 0,009108325 & $-0,018212105$ & $-0,50012478$ \\
\hline 4000 & $-0,004315441$ & 0,008606909 & $-0,50139266$ \\
\hline 5000 & 0,003783442 & $-0,007549603$ & $-0,501144497$ \\
\hline 6000 & $-0,005834998$ & 0,01166299 & $-0,500300352$ \\
\hline 7000 & 0,005184651 & $-0,010374924$ & $-0,499729058$ \\
\hline 8000 & 0,001131601 & $-0,002253457$ & $-0,502162233$ \\
\hline 9000 & $-0,005238931$ & 0,010478471 & $-0,49997094$ \\
\hline 10000 & $-0,000950073$ & 0,00189316 & $-0,501845063$ \\
\hline 20000 & $-0,0006394$ & 0,001281242 & $-0,499047018$ \\
\hline 30000 & 0,001044122 & $-0,002086959$ & $-0,500307864$ \\
\hline 40000 & 0,001316711 & $-0,002634157$ & $-0,499860487$ \\
\hline 50000 & $-0,001201397$ & 0,002403316 & $-0,4998914$ \\
\hline 60000 & 5.52E-01 & $-\mathrm{I}, 15162 \mathrm{E}-05$ & $-0,47912419$ \\
\hline 70000 & 0,001548127 & $-0,003096024$ & $-0,500037144$ \\
\hline 80000 & $-0,001414379$ & 0,002828936 & $-0,499968539$ \\
\hline 90000 & $-0,00087016$ & 0,001740092 & $-0,500065514$ \\
\hline 100000 & 0,001276813 & $-0,002553752$ & $-0,49997533$ \\
\hline 200000 & $-0,001080988$ & 0,002161994 & $-0,499995837$ \\
\hline 300000 & $-0,000629301$ & 0,001258633 & $-0,499987685$ \\
\hline 400000 & 0,00078539 & $-0,001570776$ & $-0,500001273$ \\
\hline 500000 & $-0,000701411$ & 0,001402818 & $-0,500001426$ \\
\hline 600000 & 0,000584699 & $-0,001169403$ & $-0,499997862$ \\
\hline 700000 & $-0,000101445$ & 0,000202903 & $-0,499967965$ \\
\hline 800000 & $-0,000493971$ & 0,000987937 & $-0,500002531$ \\
\hline 900000 & 0,000289383 & $-0,000578773$ & $-0,499993953$ \\
\hline 1000000 & 0,000438865 & $-0,000877726$ & $-0,500002279$ \\
\hline
\end{tabular}

Table 1: Calculation of numerical verification 1.1. 


\begin{tabular}{|c|c|c|c|}
\hline$m$ & ${ }^{R}\left(\mathbf{n}_{\mathrm{m}}\left(\mathbf{s}_{1}\right)\right)$ & $b_{m}\left(s_{1}\right)$ & $\frac{\Im\left(\eta_{m}\left(s_{1}\right)\right)}{b_{m}\left(s_{1}\right)}$ \\
\hline 10 & $0,09,94,02,356$ & $-0,28,60,65,093$ & $-0,34,74,81,599$ \\
\hline 50 & $-00,70,67,349$ & $0,13,43,53,023$ & $-0,52,60,28,283$ \\
\hline 100 & $0,04,09,08,291$ & $-0,07,71,14,071$ & $-0,53,04,90,616$ \\
\hline 150 & $0,04,07,68,907$ & $-0^{\prime} 080872098$ & $-0,50,41,15,857$ \\
\hline 200 & $-0,01,83,10,429$ & $0,03,43,93,626$ & $-0,53,23,78,558$ \\
\hline 250 & $0,01,58,37,008$ & $-0^{\prime} 030070566$ & $-0,52,66,61,454$ \\
\hline 300 & $-0^{\prime} 02553643$ & $00,50,36,579$ & $-0,50,70,19,348$ \\
\hline 350 & $0^{\prime} 023824466$ & $-0,04,80,85,954$ & $-0,49,54,55,825$ \\
\hline 400 & $0,00,38,11,944$ & $-0,00,67,44,234$ & $-0,56,52,15,264$ \\
\hline 450 & $-0,02,35,47,985$ & $0,04,71,00,327$ & $-0,49,99,53,748$ \\
\hline 500 & $-0^{\prime} 003054444$ & $0,00,54,78,744$ & $-0,55,75,08,071$ \\
\hline 550 & $0,01,99,69,559$ & $-0,04,01,06,928$ & $-0,49,79,07,967$ \\
\hline 600 & $0,01,31,42,658$ & $-00,25,90,282$ & $-0,50,73,83,289$ \\
\hline 650 & $-0,00,82,35,604$ & $0,01,68,49,851$ & $-0,48,87,64,203$ \\
\hline 700 & $-0,01,88,30,511$ & $0,03,76,77,778$ & $-0,49,97,77,641$ \\
\hline 750 & $-0,01,15,45,216$ & $0,02,28,14,158$ & $-05,06,05,488$ \\
\hline 800 & $0,00,40,06,358$ & $-0,00,83,13,703$ & $-0,48,18,98,139$ \\
\hline 850 & $0,01,51,78,428$ & $-0,03,04,78,702$ & $-0,49,80,01,129$ \\
\hline 900 & $0,01,58,06,735$ & $-0,03,15,19,545$ & $-0,50,14,89,949$ \\
\hline 950 & $0,00,75,30,681$ & $-0,01,48,42,827$ & $-0,50,73,61,637$ \\
\hline 1000 & $-0^{\prime} 003802202$ & $0,00,78,19,003$ & $-0,48,62,77,087$ \\
\hline 2000 & $00,06,48,777$ & $-0,01,30,38,104$ & $-0,49,76,00,725$ \\
\hline 3000 & $0,00,06,21,364$ & $-0,00,12,85,525$ & $-0,48,33,54,272$ \\
\hline 4000 & $-0,00,66,24,574$ & $0,01,32,63,526$ & $-0,49,94,57,987$ \\
\hline 5000 & $0,00,59,74,167$ & $-0,01,19,58,407$ & $-0,49,95,78,832$ \\
\hline 6000 & $-0^{\prime} 002760977$ & $0,00,55,35,461$ & $-0,49,87,79,957$ \\
\hline 7000 & $-0,00,29,72,586$ & $0,00,59,34,484$ & $-0,50,09,005$ \\
\hline 8000 & $0,00,54,74,619$ & $-0,01,09,50,887$ & $-0,49,99,24,709$ \\
\hline 9000 & $0,00,05,77,008$ & $-0,00,11,45,756$ & $-0,50,36,04,607$ \\
\hline 10000 & $-0,004909035$ & 0,009819162 & $-0,499944394$ \\
\hline 20000 & 0,003477281 & $-0,006954022$ & $-0,500038826$ \\
\hline 30000 & 0,002691335 & $-0,00,53,83,116$ & $-0,49,99,58,574$ \\
\hline 40000 & $-0,00,21,25,171$ & $0,00,42,49,849$ & $-0,50,00,58,002$ \\
\hline 50000 & $00,01,88,592$ & $-0,00,37,71,481$ & $-0,50,00,47,594$ \\
\hline 60000 & $-0,00,20,41,243$ & $0,00,40,82,467$ & $-0,50,00,02,327$ \\
\hline 70000 & $0,00,10,83,862$ & $-0,00,21,68,029$ & $-04,99,92,966$ \\
\hline 80000 & $0,00,10,60,449$ & $-0,00,21,20,641$ & $-0,50,00,60,595$ \\
\hline 90000 & $-0,00,14,21,483$ & 0'002843095 & $-0,49,99,77,313$ \\
\hline 100000 & $-00,00,93,261$ & $0,00,18,65,034$ & $-0,50,00,49,865$ \\
\hline 200000 & $0^{\prime} 000285425$ & $-0,00,05,70,773$ & $-0,50,00,67,452$ \\
\hline 300000 & $0,00,06,61,298$ & $-0,00,13,22,565$ & $-05,00,01,172$ \\
\hline 400000 & $9,03502 \mathrm{E}-05$ & $-0,00,01,80,728$ & $-0,49,99,23,642$ \\
\hline 500000 & $-8,95746 \mathrm{E}-05$ & $0,00,01,79,169$ & $-0,49,99,44,745$ \\
\hline 600000 & $-0,00,02,73,486$ & $0,00,05,46,957$ & $-0,50,00,13,712$ \\
\hline 700000 & $0,00,05,88,941$ & $-00,01,17,788$ & $-0,50,00,00,849$ \\
\hline 800000 & $-0,00,02,61,711$ & $0,00,05,23,431$ & $-0,49,99,91,403$ \\
\hline 900000 & $-0^{\prime} 000440494$ & $0^{\prime} 000880984$ & $-05,00,00,227$ \\
\hline 1000000 & $0,00,02,39,578$ & $-0,00,04,79,162$ & $-0,49,99,93,739$ \\
\hline
\end{tabular}

Table 2: Calculation of numerical verification 1.2 . 


\begin{tabular}{|c|c|c|c|}
\hline$m$ & ${ }^{R}\left(\eta_{m}\left(s_{2}\right)\right)$ & $a_{m}\left(s_{1}\right)$ & $\frac{\Re\left(\eta_{m}\left(s_{2}\right)\right)}{a m\left(s_{2}\right)}$ \\
\hline 10 & $-0,298803655$ & 0,090320283 & $-3,30826748$ \\
\hline 50 & 0,06833117 & $-0,120022828$ & $-0,569318113$ \\
\hline 100 & $-0,039062783$ & 0,083684493 & $-0,466786397$ \\
\hline 150 & 0,000838921 & $-0,007372696$ & $-0,113787548$ \\
\hline 200 & $-0,006958822$ & 0,01022426 & $-0,680618646$ \\
\hline 250 & $-0,030995955$ & 0,062370315 & $-0,496966466$ \\
\hline 300 & 0,025512949 & $-0,049970481$ & $-0,510560405$ \\
\hline 350 & $-0,02218031$ & 0,043391872 & $-0,511162782$ \\
\hline 400 & 0,024164864 & $-0,047925056$ & $-0,504221925$ \\
\hline 450 & $-0,021729465$ & 0,043839399 & $-0,49566065$ \\
\hline 500 & 0,005463041 & $-0,011826955$ & $-0,461914415$ \\
\hline 550 & 0,016570237 & $-0,032600321$ & $-0,508284474$ \\
\hline 600 & $-0,01650938$ & 0,033415728 & $-0,494060162$ \\
\hline 650 & $-0,009686153$ & 0,018808426 & $-0,51499009$ \\
\hline 700 & 0,016332111 & $-0,032931023$ & $-0,495949093$ \\
\hline 750 & 0,011016676 & $-0,021613651$ & $-0,509709165$ \\
\hline 800 & $-0,011529711$ & 0,023400347 & $-0,492715386$ \\
\hline 850 & $-0,015702739$ & 0,031220641 & $-0,502960173$ \\
\hline 900 & 0,000767843 & $-0,001923818$ & $-0,399124553$ \\
\hline 950 & 0,015024203 & $-0,030172433$ & $-0,497944697$ \\
\hline 1000 & 0,012184744 & $-0,024148842$ & $-0,504568459$ \\
\hline 2000 & $-0,0101168$ & 0,020280562 & $-0,498842192$ \\
\hline 3000 & 0,002094356 & $-0,004250569$ & $-0,49272368$ \\
\hline 4000 & $-2,51383 \mathrm{E}-05$ & $8.73 \mathrm{E}-01$ & $-2,8803618$ \\
\hline 5000 & $-0,007069382$ & 0,014138711 & $-0,500001874$ \\
\hline 6000 & 0,005070098 & $-0,010125745$ & $-0,500713577$ \\
\hline 7000 & $-0,00430446$ & 0,008596143 & $-0,500743182$ \\
\hline 8000 & 0,005075981 & $-0,010145473$ & $-0,500319798$ \\
\hline 9000 & $-0,005128103$ & 0,01025875 & $-0,499876008$ \\
\hline 10000 & 0,001998329 & $-0,004006189$ & $-0,498810466$ \\
\hline 20000 & 0,00234438 & $-0,004685918$ & $-0,500303249$ \\
\hline 30000 & $-0,002882405$ & 0,005764873 & $-0,499994536$ \\
\hline 40000 & $-0,002395292$ & 0,004790929 & $-0,499963994$ \\
\hline 50000 & 0,000686115 & $-0,001371329$ & $-0,500328513$ \\
\hline 60000 & 0,000755897 & $-0,001512452$ & $-0,499782472$ \\
\hline 70000 & $-0,000869896$ & 0,00174029 & $-0,49985692$ \\
\hline 80000 & 0,000253446 & $-0,000507351$ & $-0,49954765$ \\
\hline 90000 & 0,000830553 & $-0,001660765$ & $-0,500102664$ \\
\hline 100000 & $-0,001569306$ & 0,003138564 & $-0,500007647$ \\
\hline 200000 & 0,000590745 & $-0,001181589$ & $-0,499958107$ \\
\hline 300000 & 0,000308187 & $-0,000616313$ & $-0,500049488$ \\
\hline 400000 & 0,000433172 & $-0,000866309$ & $-0,500020201$ \\
\hline 500000 & 0,000583069 & $-0,001166153$ & $-0,499993569$ \\
\hline 600000 & $-0,000642894$ & 0,001285785 & $-0,500001167$ \\
\hline 700000 & 0,000586993 & $-0,001173981$ & $-0,50000213$ \\
\hline 800000 & $-0,000553097$ & 0,001106195 & $-0,499999548$ \\
\hline 900000 & 0,000362932 & $-0,000725874$ & $-0,499993112$ \\
\hline 1000000 & $8.3178 \mathrm{E}-05$ & $-0,000166333$ & $-0,500031864$ \\
\hline
\end{tabular}

Table 3: Calculation of numerical verification 1.3 . 


\begin{tabular}{|c|c|c|c|}
\hline$m$ & $\Im\left(\eta_{m}\left(s_{2}\right)\right)$ & $\mathbf{b}_{m}\left(\mathbf{s}_{2}\right)$ & $\frac{\Im\left(\eta_{m}\left(s_{2}\right)\right)}{b_{m}\left(s_{2}\right)}$ \\
\hline 10 & $-0,1292896$ & 0,303054857 & $-0,426621112$ \\
\hline 50 & 0,024809322 & $-0,074796528$ & $-0,331690824$ \\
\hline 100 & 0,031856819 & $-0,054744001$ & $-0,58192347$ \\
\hline 150 & $-0,040985555$ & 0,081316112 & $-0,50402748$ \\
\hline 200 & $-0,034758978$ & 0,069967596 & $-0,496786798$ \\
\hline 250 & 0,006559889 & $-0,010485409$ & $-0,625620708$ \\
\hline 300 & 0,01359563 & $-0,028918581$ & $-0,470134755$ \\
\hline 350 & $-0,014966219$ & 0,031213591 & $-0,479477642$ \\
\hline 400 & 0,006501864 & $-0,014254439$ & $-0,45612907$ \\
\hline 450 & 0,009181916 & $-0,017330011$ & $-0,529827477$ \\
\hline 500 & $-0,0216997$ & 0,043129145 & $-0,50313309$ \\
\hline 550 & 0,013436983 & $-0,027484557$ & $-0,488892108$ \\
\hline 600 & 0,012024249 & $-0,023453269$ & $-0,512689681$ \\
\hline 650 & $-0,017064307$ & 0,034419539 & $-0,495773839$ \\
\hline 700 & $-0,0095258$ & 0,018550449 & $-0,513507786$ \\
\hline 750 & 0,014568941 & $-0,029430994$ & $-0,495020352$ \\
\hline 800 & 0,013409517 & $-0,026503279$ & $-0,505956904$ \\
\hline 850 & $-0,006910842$ & 0,014203596 & $-0,486555799$ \\
\hline 900 & $-0,016654744$ & 0,033277771 & $-0,500476549$ \\
\hline 950 & $-0,006132047$ & 0,011927105 & $-0,514127024$ \\
\hline 1000 & 0,010083883 & $-0,020416498$ & $-0,493908554$ \\
\hline 2000 & 0,004762884 & $-0,009418005$ & $-0,505721116$ \\
\hline 3000 & $-0,008886052$ & 0,017755731 & $-0,500461062$ \\
\hline 4000 & $-0,007906176$ & 0,015811386 & $-0,500030548$ \\
\hline 5000 & 0,000170479 & $-0,00031122$ & $-0,547776493$ \\
\hline 6000 & 0,003995544 & $-0,008008493$ & $-0,498913341$ \\
\hline 7000 & $-0,004145907$ & 0,008304425 & $-0,499240706$ \\
\hline 8000 & 0,002342311 & $-0,004697805$ & $-0,498596898$ \\
\hline 9000 & 0,001217342 & $-0,002422635$ & $-0,502486755$ \\
\hline 10000 & $-0,004583444$ & 0,009162448 & $-0,500242293$ \\
\hline 20000 & 0,002646545 & $-0,005295486$ & $-0,49977377$ \\
\hline 30000 & 0,000158785 & $-0,000315547$ & $-0,503205545$ \\
\hline 40000 & 0,000716001 & $-0,001430734$ & $-0,500443129$ \\
\hline 50000 & 0,002128214 & $-0,004256695$ & $-0,499968638$ \\
\hline 60000 & $-0,001896133$ & 0,003791986 & $-0,50003692$ \\
\hline 70000 & 0,001677717 & $-0,003355161$ & $-0,500040684$ \\
\hline 80000 & $-0,00174951$ & 0,003498942 & $-0,500011146$ \\
\hline 90000 & 0,001444982 & $-0,002890151$ & $-0,499967649$ \\
\hline 100000 & $-0,000193106$ & 0,00038654 & $-0,499575723$ \\
\hline 200000 & $-0,000949222$ & 0,00189838 & $-0,500016856$ \\
\hline 300000 & 0,000859276 & $-0,001718573$ & $-0,49999389$ \\
\hline 400000 & 0,000661334 & $-0,00132269$ & $-0,499991684$ \\
\hline 500000 & $-0,000400039$ & 0,000800054 & $-0,500014999$ \\
\hline 600000 & $-5.79 \mathrm{E}+00$ & 0,000115863 & $-0,499902471$ \\
\hline 700000 & 0,000112173 & $-0,000224364$ & $-0,499959887$ \\
\hline 800000 & $8.11 \mathrm{E}+00$ & $-0,00016227$ & $-0,500046219$ \\
\hline 900000 & $-0,000382175$ & 0,000764342 & $-0,500005233$ \\
\hline 1000000 & 0,000493034 & $-0,00098607$ & $-0,499998986$ \\
\hline
\end{tabular}

Table 4: Calculation of numerical verification 1.4 . 


\begin{tabular}{|c|c|c|c|}
\hline$m$ & $\Re\left(\eta_{m}\left(s_{3}\right)\right)$ & $a_{m}\left(s_{3}\right)$ & $\frac{\Re\left(\eta_{m}\left(s_{3}\right)\right)}{\operatorname{am}\left(s_{3}\right)}$ \\
\hline 10 & 0,448284561 & $-0,159819003$ & $-2,804951555$ \\
\hline 50 & $-0,071854242$ & 0,127115345 & $-0,565268041$ \\
\hline 100 & $-0,01904003$ & 0,048915773 & $-0,389241115$ \\
\hline 150 & 0,037357774 & $-0,076882011$ & $-0,485910469$ \\
\hline 200 & 0,031022457 & $-0,059585908$ & $-0,520634124$ \\
\hline 250 & 0,031161654 & $-0,062681924$ & $-0,497139399$ \\
\hline 300 & $-0,009305261$ & 0,016286624 & $-0,57134376$ \\
\hline 350 & $-0,010228407$ & 0,02217931 & $-0,461168855$ \\
\hline 400 & 0,014023613 & $-0,029296023$ & $-0,478686578$ \\
\hline 450 & $-0,009241699$ & 0,019663369 & $-0,469995706$ \\
\hline 500 & $-0,002259912$ & 0,003402611 & $-0,664169957$ \\
\hline 550 & 0,016122602 & $-0,031579255$ & $-0,51054409$ \\
\hline 600 & $-0,019793606$ & 0,039763414 & $-0,49778437$ \\
\hline 650 & 0,003578293 & $-0,007892821$ & $-0,453360465$ \\
\hline 700 & 0,016879458 & $-0,033431943$ & $-0,504890129$ \\
\hline 750 & $-0,010664078$ & 0,021809242 & $-0,488970593$ \\
\hline 800 & $-0,013885918$ & 0,027414235 & $-0,506522177$ \\
\hline 850 & 0,009887957 & $-0,020178073$ & $-0,490034752$ \\
\hline 900 & 0,014835989 & $-0,029446734$ & $-0,503824601$ \\
\hline 950 & $-0,004109635$ & 0,008628723 & $-0,476273836$ \\
\hline 1000 & $-0,015808874$ & 0,03161724 & $-0,500008034$ \\
\hline 2000 & $-0,000363142$ & 0,000865888 & $-0,419386803$ \\
\hline 3000 & 0,006228512 & $-0,012511409$ & $-0,497826584$ \\
\hline 4000 & 0,007872022 & $-0,015738314$ & $-0,50018204$ \\
\hline 5000 & 0,005802183 & $-0,011623931$ & $-0,499158417$ \\
\hline 6000 & $-0,004449265$ & 0,008878626 & $-0,501120894$ \\
\hline 7000 & 0,000276627 & $-0,000531904$ & $-0,520069411$ \\
\hline 8000 & 0,000846807 & $-0,001710832$ & $-0,494967945$ \\
\hline 9000 & 0,000229212 & $-0,000443778$ & $-0,516501494$ \\
\hline 10000 & $-0,002612648$ & 0,005214494 & $-0,501035767$ \\
\hline 20000 & $-0,003116677$ & 0,006235361 & $-0,499839063$ \\
\hline 30000 & 0,002814184 & $-0,005627784$ & $-0,500051885$ \\
\hline 40000 & 0,001051196 & $-0,002100961$ & $-0,500340558$ \\
\hline 50000 & 0,002028101 & $-0,004055711$ & $-0,500060532$ \\
\hline 60000 & 0,000568791 & $-0,001138395$ & $-0,499642918$ \\
\hline 70000 & $-0,001586411$ & 0,003173177 & $-0,499944062$ \\
\hline 80000 & 0,001644039 & $-0,00328827$ & $-0,499970805$ \\
\hline 90000 & $-0,001401291$ & 0,002802825 & $-0,499956651$ \\
\hline 100000 & 0,000747314 & $-0,001494973$ & $-0,499884613$ \\
\hline 200000 & $-0,000953273$ & 0,00190647 & $-0,500019932$ \\
\hline 300000 & 0,000273893 & $-0,000547713$ & $-0,500066641$ \\
\hline 400000 & $-0,000451122$ & 0,000902284 & $-0,499977834$ \\
\hline 500000 & $6,70006 \mathrm{E}-05$ & $-0,000133966$ & $-0,500131377$ \\
\hline 600000 & 0,000625865 & $-0,001251737$ & $-0,499997204$ \\
\hline 700000 & $-0,000533734$ & 0,001067459 & $-0,500004216$ \\
\hline 800000 & 0,000439992 & $-0,000879973$ & $-0,50000625$ \\
\hline 900000 & $-0,000470137$ & 0,000940267 & $-0,500003722$ \\
\hline 1000000 & 0,00049965 & $-0,0009993$ & $-0,5$ \\
\hline
\end{tabular}

Table 5: Calculation of numerical verification 1.5 . 


\begin{tabular}{|c|c|c|c|}
\hline$m$ & $\Im\left(\eta_{m}\left(s_{3}\right)\right)$ & $\mathbf{b}_{\mathrm{m}}\left(\mathbf{s}_{3}\right)$ & $\frac{\Im\left(\eta_{m}\left(s_{3}\right)\right)}{b_{m}\left(s_{3}\right)}$ \\
\hline 10 & 0,063087093 & $-0,272869724$ & $-0,231198581$ \\
\hline 50 & $-0,014944382$ & 0,061981361 & $-0,241110904$ \\
\hline 100 & 0,046798444 & $-0,087219534$ & $-0,536559207$ \\
\hline 150 & $-0,016981693$ & 0,027492236 & $-0,6176905$ \\
\hline 200 & 0,017195077 & $-0,038072556$ & $-0,45163968$ \\
\hline 250 & $-0,005785644$ & 0,00842475 & $-0,686743702$ \\
\hline 300 & $-0,027378675$ & 0,055390245 & $-0,494286945$ \\
\hline 350 & 0,024730603 & $-0,048633538$ & $-0,508509231$ \\
\hline 400 & $-0,02073001$ & 0,040518429 & $-0,511619293$ \\
\hline 450 & 0,02170702 & $-0,042843601$ & $-0,506657225$ \\
\hline 500 & $-0,022264473$ & 0,044591728 & $-0,499296035$ \\
\hline 550 & 0,01397341 & $-0,02865192$ & $-0,487695414$ \\
\hline 600 & 0,005040697 & $-0,009248653$ & $-0,545019583$ \\
\hline 650 & $-0,019293777$ & 0,038420892 & $-0,502168898$ \\
\hline 700 & 0,008520338 & $-0,017631694$ & $-0,483239897$ \\
\hline 750 & 0,0148299 & $-0,02928635$ & $-0,506375837$ \\
\hline 800 & $-0,010952313$ & 0,022326212 & $-0,490558497$ \\
\hline 850 & $-0,014020797$ & 0,027736545 & $-0,505499045$ \\
\hline 900 & 0,007607843 & $-0,01562053$ & $-0,487041285$ \\
\hline 950 & 0,015698822 & $-0,031275817$ & $-0,501947623$ \\
\hline 1000 & 0,000493631 & $-0,000591706$ & $-0,834250455$ \\
\hline 2000 & 0,011176058 & $-0,022343908$ & $-0,500183674$ \\
\hline 3000 & $-0,006674901$ & 0,01329654 & $-0,502002852$ \\
\hline 4000 & 0,000734637 & $-0,001518383$ & $-0,48382852$ \\
\hline 5000 & $-0,004042275$ & 0,008055075 & $-0,501829592$ \\
\hline 6000 & $-0,004677003$ & 0,009372121 & $-0,49903357$ \\
\hline 7000 & 0,005969961 & $-0,011940445$ & $-0,4999781$ \\
\hline 8000 & $-0,005525843$ & 0,011048668 & $-0,500136578$ \\
\hline 9000 & 0,005265628 & $-0,01053158$ & $-0,499984618$ \\
\hline 10000 & $-0,004263257$ & 0,008532822 & $-0,499630368$ \\
\hline 20000 & 0,001669324 & $-0,003334707$ & $-0,500590906$ \\
\hline 30000 & 0,000643305 & $-0,001288945$ & $-0,49909422$ \\
\hline 40000 & 0,002268274 & $-0,004537176$ & $-0,499930794$ \\
\hline 50000 & 0,00094173 & $-0,001884465$ & $-0,499733346$ \\
\hline 60000 & $-0,001960402$ & 0,003920551 & $-0,500032266$ \\
\hline 70000 & 0,001027013 & $-0,002053451$ & $-0,500140008$ \\
\hline 80000 & $-0,000649735$ & 0,001298953 & $-0,500199006$ \\
\hline 90000 & 0,000902318 & $-0,00180424$ & $-0,500109741$ \\
\hline 100000 & $-0,001393389$ & 0,002786585 & $-0,50003463$ \\
\hline 200000 & $-0,000584187$ & 0,001168492 & $-0,499949508$ \\
\hline 300000 & 0,000870814 & $-0,00174165$ & $-0,499993684$ \\
\hline 400000 & 0,000649223 & $-0,001298416$ & $-0,500011553$ \\
\hline 500000 & 0,000703926 & $-0,001407854$ & $-0,49999929$ \\
\hline 600000 & $-0,000157985$ & 0,000315945 & $-0,500039564$ \\
\hline 700000 & $-0,000268832$ & 0,000537684 & $-0,499981402$ \\
\hline 800000 & 0,00034483 & $-0,000689672$ & $-0,4999913$ \\
\hline 900000 & $-0,00023822$ & 0,000476454 & $-0,499985308$ \\
\hline 1000000 & $-\mathrm{I}, 87141 \mathrm{E}-05$ & $3,74162 \mathrm{E}-05$ & $-0,500160358$ \\
\hline
\end{tabular}

Table 6: Calculation of numerical verification 1.6 . 


\begin{tabular}{|c|c|c|c|}
\hline$m$ & $\Re\left(\eta_{m}\left(s_{4}\right)\right)$ & $a_{m}\left(s_{4}\right)$ & $\frac{\Re\left(\eta_{m}\left(s_{4}\right)\right)}{a m\left(s_{4}\right)}$ \\
\hline 10 & 0,743881692 & $-0,186295877$ & $-3,993012105$ \\
\hline 50 & 0,058896264 & $-0,13246976$ & $-0,444601575$ \\
\hline 100 & $-0,008037138$ & 0,030587692 & $-0,262757255$ \\
\hline 150 & 0,000843704 & 0,006583153 & 0,12816108 \\
\hline 200 & $-0,021972495$ & 0,039421248 & $-0,557376951$ \\
\hline 250 & $-0,004620945$ & 0,005397286 & $-0,856160856$ \\
\hline 300 & $-0,022151837$ & 0,042272429 & $-0,524025648$ \\
\hline 350 & $-0,016905337$ & 0,035522259 & $-0,475908275$ \\
\hline 400 & 0,025014555 & $-0,049851106$ & $-0,501785357$ \\
\hline 450 & $-0,020871699$ & 0,040929649 & $-0,509940826$ \\
\hline 500 & 0,019049601 & $-0,037331041$ & $-0,510288502$ \\
\hline 550 & $-0,020296719$ & 0,040180244 & $-0,505141756$ \\
\hline 600 & 0,020103667 & $-0,040348224$ & $-0,498254074$ \\
\hline 650 & $-0,01246367$ & 0,02561283 & $-0,48661823$ \\
\hline 700 & $-0,003702041$ & 0,006592795 & $-0,561528305$ \\
\hline 750 & 0,017265557 & $-0,03426361$ & $-0,503903617$ \\
\hline 800 & $-0,011751598$ & 0,023989606 & $-0,489862068$ \\
\hline 850 & $-0,009247696$ & 0,017967146 & $-0,514700331$ \\
\hline 900 & 0,015357509 & $-0,030917047$ & $-0,496732725$ \\
\hline 950 & 0,005180575 & $-0,009863568$ & $-0,525223226$ \\
\hline 1000 & $-0,014941446$ & 0,03002631 & $-0,497611794$ \\
\hline 2000 & 0,003748574 & $-0,007655995$ & $-0,489625973$ \\
\hline 3000 & 0,001040701 & $-0,002173143$ & $-0,478892093$ \\
\hline 4000 & 0,004176745 & $-0,008301795$ & $-0,503113483$ \\
\hline 5000 & 0,000352638 & $-0,000662262$ & $-0,532475063$ \\
\hline 6000 & 0,004564823 & $-0,009106065$ & $-0,501294796$ \\
\hline 7000 & 0,004131647 & $-0,008281728$ & $-0,498887068$ \\
\hline 8000 & $-0,005554139$ & 0,011105475 & $-0,500126199$ \\
\hline 9000 & 0,004476607 & $-0,008943536$ & $-0,500541061$ \\
\hline 10000 & $-0,004068925$ & 0,008128786 & $-0,500557525$ \\
\hline 20000 & 0,003396641 & $-0,006794685$ & $-0,499896758$ \\
\hline 30000 & 0,002517775 & $-0,005036938$ & $-0,499862218$ \\
\hline 40000 & $-0,000945091$ & 0,00189193 & $-0,499538038$ \\
\hline 50000 & $-0,001742758$ & 0,003486351 & $-0,499880247$ \\
\hline 60000 & $-0,000320479$ & 0,000641978 & $-0,49920558$ \\
\hline 70000 & 0,001872554 & $-0,003744983$ & $-0,500016689$ \\
\hline 80000 & $-0,000869719$ & 0,001738848 & $-0,500169653$ \\
\hline 90000 & 0,000120573 & $-0,000240583$ & $-0,501170074$ \\
\hline 100000 & $-1,32844 \mathrm{E}-05$ & $2,60877 \mathrm{E}-05$ & $-0,509220821$ \\
\hline 200000 & 0,000883075 & $-0,001766044$ & $-0,500030011$ \\
\hline 300000 & 0,000829724 & $-0,001659407$ & $-0,500012354$ \\
\hline 400000 & $-0,000767542$ & 0,001535097 & $-0,499995766$ \\
\hline 500000 & $-0,000682612$ & 0,001365212 & $-0,500004395$ \\
\hline 600000 & $-0,000574894$ & 0,001149802 & $-0,4999993912$ \\
\hline 700000 & 0,0002836 & $-0,000567177$ & $-0,500020276$ \\
\hline 800000 & 0,000231324 & $-0,000462667$ & $-0,4999979467$ \\
\hline 900000 & $-0,000402352$ & 0,000804714 & $-0,499993787$ \\
\hline 1000000 & 0,000401575 & $-0,000803159$ & $-0,499994397$ \\
\hline
\end{tabular}

Table 7: Calculation of numerical verification 1.7 . 


\begin{tabular}{|c|c|c|c|}
\hline$m$ & $\mathfrak{J}\left(\eta_{m}\left(s_{4}\right)\right)$ & $b_{m}\left(s_{4}\right)$ & $\frac{\Im\left(\eta_{m}\left(s_{4}\right)\right)}{b_{m}\left(s_{4}\right)}$ \\
\hline 10 & 0,16006872 & $-0,255526606$ & -0 '626426823 \\
\hline 50 & $-0,045711611$ & 0,049515278 & $-0,923181952$ \\
\hline 100 & 0,050075594 & $-0,095207106$ & $-0,525964879$ \\
\hline 150 & 0,041096814 & $-0,081383836$ & $-0,50497514$ \\
\hline 200 & $-0,027886389$ & 0,058702344 & $-0,475047283$ \\
\hline 250 & $-0,031374863$ & 0,063014834 & $-0,497896464$ \\
\hline 300 & $-0,018605809$ & 0,039323976 & $-0,473141602$ \\
\hline 350 & 0,020757479 & $-0,039941357$ & $-0,519698893$ \\
\hline 400 & 0,000980712 & $-0,003855806$ & $-0,254346821$ \\
\hline 450 & $-0,011008302$ & 0,023387733 & $-0,470687005$ \\
\hline 500 & 0,011750631 & $-0,024625056$ & $-0,4771819$ \\
\hline 550 & $-0,006584136$ & 0,014273394 & $-0,461287343$ \\
\hline 600 & $-0,003622867$ & 0,006219928 & $-0,582461244$ \\
\hline 650 & 0,015158478 & $-0,029705967$ & $-0,510283944$ \\
\hline 700 & $-0,018543516$ & 0,037217019 & $-0,498253662$ \\
\hline 750 & 0,005966035 & $-0,012622931$ & $-0,472634684$ \\
\hline 800 & 0,013217734 & $-0,025971115$ & $-0,508939797$ \\
\hline 850 & $-0,014452169$ & 0,029217328 & $-0,494643761$ \\
\hline 900 & $-0,006492962$ & 0,012459829 & $-0,521111646$ \\
\hline 950 & 0,01537939 & $-0,030908601$ & $-0,497576387$ \\
\hline 1000 & 0,005190006 & $-0,009920722$ & $-0,523148013$ \\
\hline 2000 & $-0,010535022$ & 0,021009182 & $-0,501448462$ \\
\hline 3000 & $-0,009070078$ & 0,018127625 & $-0,500345633$ \\
\hline 4000 & 0,006712936 & $-0,013456605$ & $-0,49885807$ \\
\hline 5000 & 0,007062656 & $-0,014126621$ & $-0,499953669$ \\
\hline 6000 & 0,004564295 & $-0,009151298$ & $-0,498759302$ \\
\hline 7000 & $-0,004318159$ & 0,008618012 & $-0,501062078$ \\
\hline 8000 & $-0,000635297$ & 0,001291671 & $-0,491841189$ \\
\hline 9000 & 0,002781975 & $-0,005578913$ & $-0,498658968$ \\
\hline 10000 & $-0,002906056$ & 0,005824331 & $-0,498951038$ \\
\hline 20000 & $-0,000981404$ & 0,001957614 & $-0,501326615$ \\
\hline 30000 & $-0,001412192$ & 0,002821806 & $-0,5004568$ \\
\hline 40000 & 0,002314493 & $-0,004628239$ & $-0,5000807$ \\
\hline 50000 & 0,001401015 & $-0,002800957$ & $-0,500191542$ \\
\hline 60000 & 0,002015935 & $-0,004031691$ & $-0,500022199$ \\
\hline 70000 & 0,000254944 & $-0,000510702$ & $-0,499203058$ \\
\hline 80000 & $-0,001539029$ & 0,003078377 & $-0,499948187$ \\
\hline 90000 & 0,001662304 & $-0,00332464$ & $-0,499995187$ \\
\hline 100000 & $-0,001581087$ & 0,00316217 & $-0,500000632$ \\
\hline 200000 & 0,000685697 & $-0,001371528$ & $-0,499951149$ \\
\hline 300000 & 0,000380648 & $-0,000761381$ & $-0,49994418$ \\
\hline 400000 & 0,000189421 & $-0,000378783$ & $-0,500077881$ \\
\hline 500000 & $-0,000184504$ & 0,000369049 & $-0,499944452$ \\
\hline 600000 & 0,000293537 & $-0,000587045$ & $-0,5000247$ \\
\hline 700000 & 0,000526036 & $-0,001052084$ & $-0,499994297$ \\
\hline 800000 & $-0,00050891$ & 0,001017811 & $-0,500004421$ \\
\hline 900000 & 0,000340428 & $-0,000680842$ & $-0,500010281$ \\
\hline 1000000 & $-0,000297889$ & 0,000595765 & $-0,50001091$ \\
\hline
\end{tabular}

Table 8: Calculation of numerical verification 1.8 . 


\begin{tabular}{|c|c|c|c|}
\hline$m$ & $\Re\left(\eta_{m}\left(\mathbf{s}_{5}\right)\right)$ & $a_{m}\left(s_{5}\right)$ & $\frac{\mathfrak{R}\left(\eta_{m}\left(s_{5}\right)\right)}{a_{m}\left(s_{5}\right)}$ \\
\hline 10 & $0,96,21,75,573$ & $-0,28,64,35,644$ & $-33,59,13,352$ \\
\hline 50 & $-0^{\prime} 07204829$ & $0,14,13,22,356$ & $-0,50,98,15,234$ \\
\hline 100 & $0,03,85,10,014$ & $-0,06,40,90,757$ & $-0,60,08,66,893$ \\
\hline 150 & 0,000725118 & $00,07,49,855$ & $0,09,67,01,096$ \\
\hline 200 & $0,00,21,22,301$ & $-0,01,00,08,362$ & $-0,21,20,52,781$ \\
\hline 250 & $0,02,88,55,067$ & $-0,05,91,30,526$ & $-0,48,79,89,351$ \\
\hline 300 & $0,02,22,05,736$ & $-0 ' 046269956$ & $-0,47,99,16,947$ \\
\hline 350 & $-0,00,85,13,308$ & $0,01,45,94,976$ & $-0,58,33,04,008$ \\
\hline 400 & $-00,20,19,761$ & $0,04,15,16,874$ & $-0,48,64,91,589$ \\
\hline 450 & $0,02,34,56,676$ & $-0,04,66,35,379$ & $-05,02,98,028$ \\
\hline 500 & $-0^{\prime} 020232304$ & $00,39,77,083$ & $-0,50,87,22,197$ \\
\hline 550 & $0,01,92,83,981$ & $-0,03,79,69,523$ & $-0,50,78,80,518$ \\
\hline 600 & $-0,02,01,37,142$ & $0,04,00,38,679$ & $-0,50,29,42,217$ \\
\hline 650 & $0,01,85,40,022$ & $-0,03,73,67,727$ & $-0,49,61,50,649$ \\
\hline 700 & $-0^{\prime} 009683806$ & $0,02,01,13,366$ & $-0,48,14,61,233$ \\
\hline 750 & $-0,00,59,21,664$ & $0,01,10,75,533$ & $-0,53,46,61,763$ \\
\hline 800 & $0,01,72,37,036$ & $-0^{\prime} 034285655$ & $-0,50,27,47,753$ \\
\hline 850 & $-0,01,04,26,495$ & $0,02,13,66,588$ & $-0,48,79,81,282$ \\
\hline 900 & $-0,00,94,81,895$ & $00,18,45,065$ & $-0,51,39,05,743$ \\
\hline 950 & $0,01,49,85,393$ & $-0,03,01,69,727$ & $-04,96,70,297$ \\
\hline 1000 & $0,00,42,88,876$ & $-00,08,07,219$ & $-0,53,13,15,046$ \\
\hline 2000 & $0,00,60,46,856$ & $-0,01,22,46,231$ & $-0,49,37,72,819$ \\
\hline 3000 & $0,00,89,30,818$ & $-0^{\prime} 017880408$ & $-0,49,94,75,068$ \\
\hline 4000 & $-0^{\prime} 00780822$ & $0,01,56,25,417$ & $-0,49,97,12,744$ \\
\hline 5000 & $-0^{\prime} 004344305$ & $0,00,86,51,331$ & $-0,50,21,54,524$ \\
\hline 6000 & $-0,00,52,10,786$ & $0,01,04,00,146$ & $-0,50,10,30,082$ \\
\hline 7000 & $-0,00,50,17,103$ & $00,10,04,907$ & $-0,49,92,60,429$ \\
\hline 8000 & $0,00,43,39,471$ & $-0,00,86,64,127$ & $-0,50,08,54,962$ \\
\hline 9000 & $-0,00,07,92,091$ & $0,00,15,65,065$ & $-0,50,61,07,414$ \\
\hline 10000 & $-0,000884296$ & 0,00178475 & $-0,495473316$ \\
\hline 20000 & 0,003005846 & $-0,006014678$ & $-0,499751774$ \\
\hline 30000 & 0,002808479 & $-0,005616177$ & $-0,500069531$ \\
\hline 40000 & $-0,002400136$ & 0,004799665 & -500063234 \\
\hline 50000 & $-0,000490229$ & 0,000979016 & $-0,500736454$ \\
\hline 60000 & $-0,000977535$ & 0,001954077 & $-0,500254084$ \\
\hline 70000 & $-0,001872691$ & 0,003745487 & $-0,499985983$ \\
\hline 80000 & 0,000767762 & $-0,001534864$ & $-0,500215003$ \\
\hline 90000 & 0,000473951 & 0,000948484 & $-0,499693195$ \\
\hline 100000 & $-0,000914615$ & 0,001829651 & $-0,499884951$ \\
\hline 200000 & 0,001110497 & $-0,002221013$ & $-0,499995723$ \\
\hline 300000 & 0,000714685 & $-0,001429306$ & $-0,50022388$ \\
\hline 400000 & $-0,000593546$ & 0,001187049 & $-0,500018112$ \\
\hline 500000 & 0,000152119 & $-0,000304283$ & $-0,499926056$ \\
\hline 600000 & $-3,79 \mathrm{E}-05$ & 7,94E-05 & $-0,500222865$ \\
\hline 700000 & $-0,000570392$ & 0,0011400775 & $-0,500003945$ \\
\hline 800000 & $6,44 \mathrm{E}-06$ & $-1,29 \mathrm{E}-05$ & $-0,500888333$ \\
\hline 900000 & 0,000349926 & $-0,000699866$ & $-0,499989998$ \\
\hline 1000000 & $-0,000434836$ & 0,000869679 & $-0,499995976$ \\
\hline
\end{tabular}

Table 9: Calculation of numerical verification 1.9 . 


\begin{tabular}{|c|c|c|c|}
\hline$m$ & $\Im\left(\eta_{m}\left(s_{5}\right)\right)$ & $\mathbf{b}_{\mathrm{m}}\left(\mathbf{s}_{5}\right)$ & $\frac{\Im\left(\eta_{m}\left(s_{5}\right)\right)}{b_{m}\left(s_{5}\right)}$ \\
\hline 10 & -0 '099279099 & $-0,13,39,94,857$ & 0,740917235 \\
\hline 50 & $0,02,14,81,704$ & $0,00,52,90,727$ & $4,06,02,55,613$ \\
\hline 100 & $00,33,15,987$ & $-0,07,67,61,806$ & $-0,43,19,83,974$ \\
\hline 150 & $0,04,11,35,797$ & $-0,08,13,04,603$ & $-0,50,59,46,717$ \\
\hline 200 & $-00,35,45,701$ & 0'069998805 & $-0,50,65,37,362$ \\
\hline 250 & $-0,01,31,81,946$ & $0,02,24,40,609$ & $-0,58,74,14,807$ \\
\hline 300 & $-00,18,55,146$ & $0,03,45,31,501$ & $-0,53,72,32,946$ \\
\hline 350 & $-0^{\prime} 025385438$ & $0,05,14,211$ & $-0,49,36,77,459$ \\
\hline 400 & $0,01,47,95,353$ & $-0,02,78,63,043$ & $-05,31,00,277$ \\
\hline 450 & $0,00,25,89,303$ & $-0,00,68,82,125$ & $-0,37,62,35,974$ \\
\hline 500 & $-0,00,95,75,928$ & 0,020451922 & $-0,46,82,16,532$ \\
\hline 550 & $0,00,91,37,597$ & $-0,01,94,03,534$ & $-0,47,09,24,369$ \\
\hline 600 & $-0,00,34,38,645$ & $0,00,79,73,131$ & $-04,31,27,913$ \\
\hline 650 & $-0,006436337$ & $0,01,19,21,178$ & $-0,53,99,07,801$ \\
\hline 700 & $0,01,62,42,538$ & $-0,03,20,00,374$ & $-05,07,57,338$ \\
\hline 750 & $-00,17,28,151$ & $0,03,47,94,624$ & $-0,49,66,71,842$ \\
\hline 800 & $0,00,39,63,916$ & $-0^{\prime} 008630982$ & $-0,45,92,65,933$ \\
\hline 850 & $0,01,36,26,793$ & $-0,02,68,31,688$ & $-0,50,78,61,935$ \\
\hline 900 & $-0,01,37,15,647$ & $0,02,77,61,207$ & $-0,49,40,58,021$ \\
\hline 950 & $-0^{\prime} 006230058$ & $00,11,93,395$ & $-0,52,20,44,922$ \\
\hline 1000 & $0,01,52,24,928$ & $-0,03,05,75,149$ & $-0,49,79,51,065$ \\
\hline 2000 & $-00,09,40,613$ & $0,01,87,09,084$ & $-0,50,27,57,377$ \\
\hline 3000 & $-0,00,18,94,788$ & $0,00,36,91,117$ & $-0,51,33,37,291$ \\
\hline 4000 & $0,00,12,412$ & $-0,00,24,17,921$ & $-0,51,33,33,562$ \\
\hline 5000 & $-0,00,55,79,657$ & $0,01,11,87,246$ & $-0,49,87,51,614$ \\
\hline 6000 & $-0,00,38,10,271$ & $0,00,76,48,766$ & $-0,49,81,54,997$ \\
\hline 7000 & $0,00,32,47,415$ & $-00,06,47,096$ & $-0,50,18,44,394$ \\
\hline 8000 & $0,00,35,24,353$ & $-0^{\prime} 007066322$ & $-0,49,87,53,524$ \\
\hline 9000 & $-0,00,52,10,759$ & 0'010424091 & $-0,49,98,76,584$ \\
\hline 100000 & 0,001289763 & $-0,000259037$ & $-0,50002879$ \\
\hline 20000 & $-0,001861508$ & 0,003718016 & $-0,500672402$ \\
\hline 30000 & $0,00,06,67,771$ & $-0,00,13,38,615$ & $-0,49,88,52,172$ \\
\hline 40000 & $-0,00,06,99,591$ & $0,00,14,01,147$ & $-0,49,92,98,789$ \\
\hline 50000 & $-00,02,18,168$ & $00,04,36,366$ & $-0,49,99,65,625$ \\
\hline 60000 & $-0,00,17,91,963$ & $0,00,35,84,445$ & $-0,49,99,27,604$ \\
\hline 70000 & $0,00,02,53,936$ & $-00,00,50,699$ & $-05,00,86,984$ \\
\hline 80000 & $0,00,15,92,344$ & $-0,00,31,84,995$ & $-0,499951805$ \\
\hline 90000 & $-0,00,15,97,863$ & $0,00,31,95,542$ & $-05,00,02,879$ \\
\hline 100000 & $0,00,12,89,763$ & $-0^{\prime} 00257922$ & $-05,00,05,932$ \\
\hline 200000 & $-0,00,01,29,611$ & $0,00,02,59,037$ & $-0,50,03,57,092$ \\
\hline 300000 & 0'000567943 & $-0,00,11,35,966$ & $-0,49,99,64,788$ \\
\hline 400000 & $-0,00,05,22,211$ & $0,00,10,44,469$ & $-0,49,99,77,501$ \\
\hline 500000 & $-0^{\prime} 000690552$ & $0,00,13,81,091$ & $-0,50,00,04,706$ \\
\hline 600000 & $-0,00,06,44,275$ & $0,00,12,88,549$ & $-0,50,00,00,388$ \\
\hline 700000 & $-0,00,01,78,315$ & $0^{\prime} 000356656$ & $-04,99,96,355$ \\
\hline 800000 & $0,00,05,58,979$ & $-00,01,11,796$ & $-0,49,99,99,106$ \\
\hline 900000 & $-00,00,39,412$ & 0'000788225 & $-0,50,00,09,515$ \\
\hline 1000000 & $0^{\prime} 000246815$ & $-0,00,04,93,617$ & $-0,50,00,13,168$ \\
\hline
\end{tabular}

Table 10: Calculation of numerical verification 1.10 


\begin{tabular}{|c|c|c|c|}
\hline$m$ & $\mathfrak{R}\left(\eta_{m}\left(s^{\prime}\right)\right)$ & $a_{m}\left(s_{1}^{\prime}\right)$ & $\frac{\Re\left(\eta_{m}\left(s_{1}^{\prime}\right)\right)}{\operatorname{am}\left(s_{1}^{\prime}\right)}$ \\
\hline 10 & $-0,001870233$ & 0,043670938 & $-0,042825574$ \\
\hline 50 & $-0,006962498$ & 0,012371397 & $-0,562789958$ \\
\hline 100 & $-0,003286542$ & 0,006185698 & $-0,531313039$ \\
\hline 150 & 0,000543644 & $-0,000884624$ & $-0,614548102$ \\
\hline 200 & $-0,001594816$ & 0,003092849 & $-0,515646254$ \\
\hline 250 & 0,001950134 & $-0,00390792$ & $-0,499020963$ \\
\hline 300 & 0,000246483 & $-0,000442312$ & $-0,557260486$ \\
\hline 350 & $-0,001358336$ & 0,002723944 & $-0,498665171$ \\
\hline 400 & $-0,000785308$ & 0,001546425 & $-0,507821589$ \\
\hline 450 & 0,000423631 & $-0,000866907$ & $-0,488669488$ \\
\hline 500 & 0,000976023 & $-0,00195396$ & $-0,499510225$ \\
\hline 550 & 0,000729462 & $-0,001448551$ & $-0,503580475$ \\
\hline 600 & 0,00011691 & $-0,000221156$ & $-0,528631373$ \\
\hline 650 & $-0,000425434$ & 0,000859106 & $-0,495205481$ \\
\hline 700 & $-0,000680077$ & 0,001361972 & $-0,499332585$ \\
\hline 750 & $-0,000634749$ & 0,001266128 & $-0,501330829$ \\
\hline 800 & $-0,00038963$ & 0,000773212 & $-0,503910958$ \\
\hline 850 & $-7,20 \mathrm{E}-05$ & 0,000137773 & $-0,522902165$ \\
\hline 900 & 0,000214271 & $-0,000433453$ & $-0,494335026$ \\
\hline 950 & 0,000407904 & $-0,000818535$ & $-0,498334219$ \\
\hline 1000 & 0,000488251 & $-0,00097698$ & $-0,499755369$ \\
\hline 2000 & 0,000244185 & $-0,00048849$ & $-0,499877173$ \\
\hline 3000 & $-0,000158371$ & 0,000316532 & $-0,50033172$ \\
\hline 4000 & 0,000122108 & $-0,000244245$ & $-0,499940633$ \\
\hline 5000 & $-2,34 \mathrm{E}-05$ & $4,69 \mathrm{E}-05$ & $-0,498173531$ \\
\hline 6000 & $-7,92 \mathrm{E}-05$ & 0,000158266 & $-0,500166176$ \\
\hline 7000 & I,03E-05 & $-2,07 \mathrm{E}-05$ & $-0,49782573$ \\
\hline 8000 & $6, \mathrm{IIE}-05$ & $-0,000122122$ & $-0,49997134$ \\
\hline 9000 & 3,66E-05 & $-7,31 \mathrm{E}-05$ & $-0,50031666$ \\
\hline 10000 & $-1,17 \mathrm{E}-05$ & $2,35 \mathrm{E}-05$ & $-0,499086235$ \\
\hline 20000 & $-5,86 \mathrm{E}-06$ & I, I7E-05 & $-0,499545032$ \\
\hline 30000 & I, 16E-05 & $-2,32 \mathrm{E}-05$ & $-0,499931104$ \\
\hline 40000 & $-2,93 \mathrm{E}-06$ & 5,87E-06 & $-0,499770813$ \\
\hline 50000 & $-7,72 \mathrm{E}-06$ & I,54E-05 & $-0,500043397$ \\
\hline 60000 & 5,81E-06 & -I,16E-05 & $-0,499964691$ \\
\hline 70000 & 5,91E-06 & -I,18E-05 & $-0,500027091$ \\
\hline 80000 & -|,47E-06 & 2,93E-06 & $-0,499887536$ \\
\hline 90000 & $-5,36 \mathrm{E}-06$ & I,07E-05 & $-0,499997201$ \\
\hline 100000 & $-3,86 E-06$ & 7,72E-06 & $-0,500021374$ \\
\hline 200000 & -I,93E-06 & 3,86E-06 & $-0,500010363$ \\
\hline 300000 & $5,68 \mathrm{E}-07$ & -I,14E-06 & $-0,50002023$ \\
\hline 400000 & $-9,65 \mathrm{E}-07$ & 1,93E-06 & $-0,500004664$ \\
\hline 500000 & 9,09E-07 & -I,82E-06 & $-0,4999978$ \\
\hline 600000 & 2,84E-07 & $-5,68 \mathrm{E}-07$ & $-0,500011434$ \\
\hline 700000 & $-6,19 \mathrm{E}-07$ & I,24E-06 & $-0,499999193$ \\
\hline 800000 & $-4,82 \mathrm{E}-07$ & $9,65 \mathrm{E}-07$ & $-0,500002591$ \\
\hline 900000 & I,03E-07 & $-2,05 \mathrm{E}-07$ & $-0,499987818$ \\
\hline 1000000 & 4,54E-07 & $-9,09 E-07$ & $-0,49999945$ \\
\hline
\end{tabular}

Table 11: Calculation of numerical verification 1.11 


\begin{tabular}{|c|c|c|c|}
\hline$m$ & $\Im\left(\eta_{m}\left(s_{1}^{\prime}\right)\right)$ & $b_{m}\left(s_{1}^{\prime}\right)$ & $\frac{\Im\left(\eta_{m}\left(s_{1}^{\prime}\right)\right)}{a m\left(s_{1}^{\prime}\right)}$ \\
\hline 10 & $0,05,87,75,434$ & $-0,08,99,60,265$ & $-0,65,33,48,831$ \\
\hline 50 & $-0,00,73,75,109$ & $0,01,57,14,596$ & $-0,46,93,15,851$ \\
\hline 100 & $-00,03,80,811$ & $0,00,78,57,298$ & $-0,48,46,58,976$ \\
\hline 150 & $0,00,33,01,521$ & $-0,006607714$ & $-0,49,96,46,474$ \\
\hline 200 & $-00,01,93,419$ & $0,00,39,28,649$ & $-0,49,23,29,551$ \\
\hline 250 & $-0,00,04,62,944$ & $0,00,08,53,326$ & $-0,54,25,17,162$ \\
\hline 300 & $0,00,16,51,342$ & $-0,00,33,03,857$ & $-04,99,82,248$ \\
\hline 350 & $0,00,04,49,355$ & $-0,00,08,62,203$ & $-0,52,11,70,768$ \\
\hline 400 & $-0,00,09,74,629$ & $0,00,19,64,325$ & $-04,96,16,484$ \\
\hline 450 & $-00,01,02,858$ & $0,00,20,46,153$ & $-0,50,26,89,682$ \\
\hline 500 & $-0) 000222401$ & $0,00,04,26,663$ & $-0,52,12,56,823$ \\
\hline 550 & $0,00,05,43,959$ & $-0,00,10,98,857$ & $-0,49,50,22,555$ \\
\hline 600 & $0,00,08,25,817$ & $-0,00,16,51,929$ & $-04,99,91,071$ \\
\hline 650 & $0,00,06,41,609$ & $-0,00,12,76,245$ & $-0,50,27,31,842$ \\
\hline 700 & $0,00,02,20,114$ & $-0,00,04,31,101$ & $-0,51,05,85,686$ \\
\hline 750 & $-0,00,02,05,298$ & $0,00,04,17,968$ & $-0,49,11,81,143$ \\
\hline 800 & $-0,00,04,89,198$ & $0,00,09,82,162$ & $-0,49,80,82,801$ \\
\hline 850 & $-5,84 \mathrm{E}-04$ & $0,00,11,68,376$ & $-0,49,99,79,459$ \\
\hline 900 & $-0,00,05,12,914$ & $0,00,10,23,077$ & $-0,50,13,44,474$ \\
\hline 950 & $-0,00,03,33,047$ & $0,00,06,61,841$ & $-0,50,32,13,007$ \\
\hline 1000 & $-0,00,01,08,933$ & $0,00,02,13,331$ & $-0,51,06,29,023$ \\
\hline 2000 & $-5,38997 \mathrm{E}-05$ & $0,00,01,06,666$ & $-0,50,53,12,846$ \\
\hline 3000 & $-5,20156 \mathrm{E}-05$ & $0,00,01,04,492$ & $-0,49,77,95,047$ \\
\hline 4000 & $-2,68081 \mathrm{E}-05$ & $5,33329 \mathrm{E}-05$ & $-0,50,26,55,959$ \\
\hline 5000 & $9,72 \mathrm{E}-05$ & $-1,94 \mathrm{E}-04$ & $-0,50,01,58,426$ \\
\hline 6000 & -2) 61E-05 & $0,00,00,52,246$ & $-0,49,88,97,523$ \\
\hline 7000 & $-7,07 \mathrm{E}-05$ & $1,41 \mathrm{E}-04$ & $-0,50,00,82,773$ \\
\hline 8000 & $-1,34 \mathrm{E}-05$ & 2,66664E-05 & $-0,50,13,27,513$ \\
\hline 9000 & $4,18 \mathrm{E}-05$ & $-8,37 E-05$ & $-04,99,80,818$ \\
\hline 10000 & 4,86E-05 & $-9,72 \mathrm{E}-05$ & $-0,50,00,79,213$ \\
\hline 20000 & $2,43 \mathrm{E}-05$ & $-4,86 \mathrm{E}-05$ & $-0,50,00,40,121$ \\
\hline 30000 & $-1,20 \mathrm{E}-05$ & $2,39 \mathrm{E}-05$ & $-05,00,08,364$ \\
\hline 40000 & I,22E-05 & $-2,43 E-05$ & $-0,50,00,20,575$ \\
\hline 50000 & $-6,36 \mathrm{E}-06$ & I,27E-05 & $-0,49,99,51,233$ \\
\hline 60000 & $-5,98 \mathrm{E}-06$ & $1,20 \mathrm{E}-05$ & $-05,00,04,182$ \\
\hline 70000 & $4,02 \mathrm{E}-06$ & $-8,03 E-06$ & $-0,49,99,56,439$ \\
\hline 80000 & $6,08 \mathrm{E}-06$ & $-1,22 \mathrm{E}-05$ & $-0,50,00,10,699$ \\
\hline 90000 & I,46E-06 & $-2,92 \mathrm{E}-06$ & $-0,50,00,94,092$ \\
\hline 100000 & $-3,18 \mathrm{E}-06$ & $6,36 \mathrm{E}-06$ & $-04,99,97,483$ \\
\hline 200000 & $-1,59 \mathrm{E}-06$ & $3,18 \mathrm{E}-06$ & $-0,49,99,85,842$ \\
\hline 300000 & 1,57E-06 & $-3,13 \mathrm{E}-06$ & -05 \\
\hline 400000 & $-7,95 \mathrm{E}-07$ & I,59E-06 & $-0,49,99,94,966$ \\
\hline 500000 & $-4,17 \mathrm{E}-07$ & $8,34 \mathrm{E}-07$ & $-0,50,00,10,795$ \\
\hline 600000 & 7,83E-07 & $-1,57 \mathrm{E}-06$ & $-0,49,99,99,362$ \\
\hline 700000 & $3,56 \mathrm{E}-07$ & $-7,12 \mathrm{E}-07$ & $-0,50,00,06,323$ \\
\hline 800000 & $-3,97 \mathrm{E}-07$ & $7,95 \mathrm{E}-07$ & $-0,49,99,97,483$ \\
\hline 900000 & $-5,46 \mathrm{E}-07$ & $1,09 \mathrm{E}-06$ & $-0,50,00,02,747$ \\
\hline 1000000 & $-2,08 \mathrm{E}-07$ & 4,17E-07 & $-0,50,00,05,997$ \\
\hline
\end{tabular}

Table 12: Calculation of numerical verification 1.12 . 


\begin{tabular}{|c|c|c|}
\hline$m$ & $\frac{\eta_{m}\left(s_{1}\right)}{\eta_{m}\left(s_{1}^{\prime}\right)}$ & $\left|\frac{\alpha_{m}\left(s_{1}\right)}{\alpha_{m}\left(s_{1}^{\prime}\right)}\right|$ \\
\hline 10 & 3,689741821 & 3,162277858 \\
\hline 50 & 7078600484 & 7,071076839 \\
\hline 100 & 9,98959317 & 99,99995 \\
\hline 150 & 12,23517792 & 12,24744686 \\
\hline 200 & 14,12972581 & 14,142136 \\
\hline 250 & $15^{\prime} 79934786$ & 15,81130597 \\
\hline 300 & 17,30893587 & 17,32048648 \\
\hline 350 & 18,69717121 & 18,70825462 \\
\hline 400 & 19,9893288 & $2,00,00,048$ \\
\hline 450 & 21,20294133 & 21,21320595 \\
\hline 500 & 22,35083149 & 22,3605654 \\
\hline 550 & 23,44257231 & 23,4520597 \\
\hline 600 & 24,4857063 & 24,4948882 \\
\hline 650 & 26,44885426 & 25,49512053 \\
\hline 700 & 27,377337 & 26,45730969 \\
\hline 750 & 28,27604478 & 27,38598194 \\
\hline 800 & 28,27604478 & 28,2842539 \\
\hline 850 & 29,14678157 & 29,15478354 \\
\hline 900 & 29,99220262 & 29,999915 \\
\hline 950 & 30,8144809 & 30,82205544 \\
\hline 1000 & 31,61533501 & 31,62277842 \\
\hline 2000 & 44,71598015 & 44,72157446 \\
\hline 3000 & 54,76782958 & 54,77287894 \\
\hline 4000 & 63,24135589 & 63,24535762 \\
\hline 5000 & 70,70715187 & 70,71052332 \\
\hline 6000 & 77,45658376 & 77,4598321 \\
\hline 7000 & 83,66307805 & 83,66605719 \\
\hline 8000 & 89,44000195 & 89,44244237 \\
\hline 9000 & 94,86559748 & 94,86848147 \\
\hline 10000 & 99,99764024 & $99,99,995$ \\
\hline 20000 & 141,4195571 & $14,14,214$ \\
\hline 30000 & 173,2036131 & 173,2047748 \\
\hline 40000 & 199,2429627 & 200,0112009 \\
\hline 50000 & 223,5514486 & 223,7048923 \\
\hline 60000 & 244,9163998 & 244,4861809 \\
\hline 70000 & 264,5124602 & 264,6271471 \\
\hline 80000 & 282,6427349 & 281,7625014 \\
\hline 90000 & 300,0219743 & 300,024477 \\
\hline 100000 & 316,1559247 & 316,1638253 \\
\hline 200000 & 447,1197902 & 447,1210085 \\
\hline 300000 & 546,7081404 & 548,252966 \\
\hline 400000 & 632,3240857 & 632,3295467 \\
\hline 500000 & 707,0654563 & 707,0558613 \\
\hline 600000 & 774,9132585 & 773,1643492 \\
\hline 700000 & 836,577005 & 836,5733484 \\
\hline 800000 & 894,7034584 & 894,2423084 \\
\hline 900000 & 948,6862736 & 950,3575028 \\
\hline 1000000 & 1000,352186 & 999,9355062 \\
\hline
\end{tabular}

Table 13: Calculation of numerical verification 1.13 
like all other numerical methods when the primitive off is not obvious by algebraic computation (Figure 2) $[7,8]$.

\section{Hypothesis}

- It is assumed here that the function- $\operatorname{arc}\left(\mathrm{M}_{\mathrm{i}} \mathrm{M}_{\mathrm{i}+1}\right)$ is concave on $\left[\mathrm{x}_{\mathrm{i}}, \mathrm{X}_{\mathrm{i}+1}\right]$,

- $(\mathrm{H} \mathrm{E})$ is the elliptical half-arc passing through the points $\mathrm{Mi}, \mathrm{M} ;+1$ and $P_{i}$; with: $N_{i}$ is the point of intersection of the curve-arc $\left(M_{i} M_{i+1}\right)$ and the vertical line whose equation is $\mathrm{x}=\mathrm{m}_{\mathrm{i}}$ where $m_{i}=\frac{x_{i}+x_{i+1}}{2}$

- $\mathrm{P}_{\mathrm{i}}$ is the orthogonal projection of $\mathrm{N}_{\mathrm{i}}$ on the axis $\mathrm{Y}$,

- $\mathrm{X}$ is the axis passing through the points $\mathrm{M}_{\mathrm{i}}$ and $\mathrm{M}_{\mathrm{i}+1}$ and $X \perp Y$ where $\mathrm{X}$ and $\mathrm{Y}$ are the two axes of the half-ellipse $(\mathrm{H} \mathrm{E})$,

- $\mathrm{a}_{\mathrm{e}}$ and $\mathrm{b}_{\mathrm{e}}$ are the two parameters of the half-ellipse (H E),

- $O^{\prime}\left(m_{i} \frac{f\left(x_{i}\right)+f\left(x_{i+1}\right)}{2}\right)$ is the origin of the reference $\left(O^{\prime}, \vec{X}, \vec{Y}\right)$,

- $\mathrm{Q}$ is the point whose coordinates $\mathrm{x}=\mathrm{X}_{\mathrm{i}+\mathrm{i}}$ and $\mathrm{y}=\mathrm{f}\left(\mathrm{x}_{\mathrm{i}}\right)$,

- $\alpha=M_{i+1} \hat{M}_{i} Q$ and $\beta=N i \hat{O}^{\prime} P_{i}$,

- $c=\left|\overrightarrow{N_{i} P_{i}}\right|$ and $d=\left(f\left(m_{i}\right)-\frac{f\left(x_{i}\right)+f\left(x_{i+1}\right)}{2}\right)$,

Calculation of the parameters $a_{e}$ and $b_{e}$ of the half-ellipse (HE): According to Pythagoras ' theorem, we have:

$$
\left(2 a_{e}\right)^{2}=\left(x_{i+1}-x_{i}\right)^{2}+\left(f\left(x_{i}+1\right)-f\left(x_{i}\right)\right)^{2}
$$

we denote: $x_{i+1}=x_{i}=h=\frac{b-a}{n}$ with $\mathrm{n}$ is the number of subintervals obtained by regular subdivision of the interval $[a, b]$, then we get: $a_{e}=\frac{\left(h^{2}+\left(f\left(x_{i}+1\right)-f\left(x_{i}\right)\right)^{2}\right)^{0.5}}{2}$

According to Pythagoras' theorem, we also have: $\mathrm{d}^{2}=\mathrm{b}^{2}+\mathrm{c}^{2}$, that is to say be $=\left(d^{2}-c^{2}\right)^{0.5}$ with:

Noticingthat $\alpha=\beta$, wecanwrite $\sin \beta=\frac{c}{d}=\sin \alpha=\frac{f\left(x_{i}+1\right)-f\left(x_{i}\right)}{2 a_{e}}$

This implies $c=d\left(\frac{f\left(x_{i}+1\right)-f\left(x_{i}\right)}{2 a_{e}}\right)$

So, $b_{e}=d\left(1-\left(\frac{f\left(x_{i}+1\right)-f\left(x_{i}\right)}{2 a_{e}}\right)^{2}\right)^{0.5}=\left(f\left(m_{i}\right)-\frac{f\left(x_{i}+1\right)-f\left(x_{i}\right)}{2}\right) \frac{h}{\left(h^{2}+\left(f\left(x_{i}+1\right)-f\left(x_{i}\right)\right)^{2}\right)^{0.5}}$

Approximate calculation of $\int_{x}^{x_{i+1}} f(x) d x$ :

$$
\begin{aligned}
& \text { Let, } s=\frac{\pi a_{e} b_{e}}{2}=\frac{\pi h}{4}\left(f\left(m_{i}\right)-\frac{f\left(x_{i}\right)+f\left(x_{i+1}\right)}{2}\right) \\
& \int_{x_{i}}^{x_{i+1}} f(x) d x \approx h\left(\frac{f\left(x_{i}\right)-f\left(x_{i+1}\right)}{2}\right)+s=\left(1-\frac{\pi}{4}\right) h\left(\frac{f\left(x_{i}\right)-f\left(x_{i+1}\right)}{2}\right)+\left(\frac{\pi}{4}\right) h f\left(m_{i}\right)
\end{aligned}
$$

\section{Appoximation formula}

Doing the summation for $\mathrm{i}$ ranging from 0 to $\mathrm{n}-1$ with $\mathrm{x}_{0}=\mathrm{a}$ and $\mathrm{x}_{\mathrm{n}}=\mathrm{b}$, we obtain the following approximation formula:

$$
\int_{a}^{b} f(x) d x \approx T H E=\left(1-\frac{\pi}{4}\right) h\left(\frac{f(a)+f(b)}{2}+\sum_{i=1}^{n-1} f\left(x_{i}\right)\right)+\left(\frac{\pi}{4}\right) h \sum_{i=0}^{n-1} f\left(m_{i}\right)
$$

Note: $h\left(\frac{f(a)+f(b)}{2}+\sum_{i=1}^{n-1} f\left(x_{i}\right)\right)=T_{n}$ The approximate value of the integral of on $[a, b]$ using the method of trapezes.

$h \sum_{i=0}^{n-1} f\left(m_{i}\right)=M_{n}:$ The approximate value of the integral of $\mathrm{f}$ on [a,b] using the method of rectangles with midpoint.

So, the approximation formula of The Method of trapezes 8 halfellipses is:

$$
\int_{a}^{b} f(x) d x \approx T H E_{n}=\left(1-\frac{\pi}{4}\right) T_{n}+\left(\frac{\pi}{4}\right) M_{n}
$$

Note: Knowing that $2 \mathrm{~T}_{2 \mathrm{n}}=\mathrm{T}_{\mathrm{n}}+\mathrm{M}_{\mathrm{n}}$, we can write: $T H E_{n}=\left(1-\frac{\pi}{2}\right) T_{n}+\left(\frac{\pi}{2}\right) T_{2 n}$

\section{Calculation of the theoretical maximum error $\mathrm{e}(\mathrm{n})$}

Let's call $e_{i}(n)$ the error committed in the subinterval $\left[\mathrm{x}_{\mathrm{i}}, \mathrm{X}_{\mathrm{i}+1}\right]$. Thus, according to the approximation formula previously established, we have:

$$
\begin{aligned}
& e_{i}(n)=\left(1-\frac{\pi}{4}\right) h\left(\frac{f\left(x_{i}\right)+f\left(x_{i+1}\right)}{2}\right)+\left(\frac{\pi}{4}\right) h f\left(m_{i}\right)-\int_{x_{i}}^{x_{i+1}} f(x) d x \\
& \left(1-\frac{\pi}{4}\right)\left(h\left(\frac{f\left(x_{i}\right)+f\left(x_{i+1}\right)}{2}\right)-\int_{x_{i}}^{x_{i+1}} f(x) d x\right)+\left(\frac{\pi}{4}\right)\left(h f\left(m_{i}\right)-\int_{x_{i}}^{x_{i+1}} f(x) d x\right) \\
& \text { Note: }\left(h\left(\frac{f\left(x_{i}\right)+f\left(x_{i+1}\right)}{2}\right)-\int_{x_{i}}^{x_{i+1}} f(x) d x\right)=e_{i / \text { trapeze }} \text { The error }
\end{aligned}
$$
committed in $\left[\mathrm{x}_{\mathrm{i}}, \mathrm{x}_{\mathrm{i}+1}\right]$ using the $\left(h f\left(m_{i}\right)-\int_{x}^{x_{i+1}} f(x) d x\right)=e_{i / \text { rec tangle }}$ : The error committed in $\left[\mathrm{x}_{\mathrm{i}}, \mathrm{x}_{\mathrm{i}+1}\right]$ using the method of rectangles with midpoint, so:

$$
e_{i / \text { trapeze }}=\left(1-\frac{\pi}{4}\right) e_{i / \text { trapeze }}+\left(\frac{\pi}{4}\right) e_{i / \text { rec tangle }}
$$
$003 \mathrm{~A}$.

We assume that $\mathrm{f}$ is class $\mathrm{C}^{2}$ on the interval $[\mathrm{a}, \mathrm{b}]$, so we know that $\left|e_{i / \text { trapeze }}\right| \leq \frac{h^{3} \lambda_{i}}{12}$ and $\left|e_{i / \text { rec tangle }}\right| \leq \frac{h^{3} \lambda_{i}}{12} \quad$ where $\quad \lambda_{i}=\sup \left|f^{\prime \prime}(u)\right|, u \in\left[x_{i}, x_{i+1}\right]$ $\mathrm{Xi}+\mathrm{i}]$, then we have:

$$
|e(n)| \leq\left(1-\frac{\pi}{8}\right) \frac{h^{3} \lambda_{i}}{12},
$$

and denoting $\lambda=\sup \left(\lambda_{i}\right)=\sup \left|f^{\prime \prime}(x)\right|, \mathrm{x} \in[a, b]$, we get:

$$
|e(n)| \leq\left(1-\frac{\pi}{8}\right) \frac{(b-a)^{3} \lambda}{12}
$$

Note: if someone replace the elliptical half-arc studied in the previous case by the quarter of the circumference of the ellipse whose parameters are $h=\frac{b-a}{n}$ and $\delta_{i}=f\left(x_{i+1}\right)-f\left(x_{i}\right)$ he gets:

$\int_{a}^{b} f(x) d x \approx R Q E_{n}=\left(1-\frac{\pi}{4}\right) R_{n}^{(\ell)}+\left(\frac{\pi}{4}\right) R_{n}^{(r)}, \quad[\mathrm{RQE}:$ Rectangles and Quarters of Ellipses].

where: 
- $\mathrm{R}_{\mathrm{n}}^{(1)}$ is the approximate value of the integral of on [a, b] using the method of rectangles on the left,

- $\mathrm{R}_{\mathrm{n}}^{(\mathrm{r})}$ is the approximate value of the integral of on $[\mathrm{a}, \mathrm{b}] \mathrm{using}$ the method of rectangles on the right,

and assuming that $\mathrm{f}$ is class $\mathrm{C}^{1}$ on the interval $[\mathrm{a}, \mathrm{b}]$, we get:

$\left|\int_{a}^{b} f(x) d x-R Q E_{n}\right| \leq \frac{(b-a)^{2}}{2} \sup _{[a, b]}\left|f^{\prime}\right|$

\section{Conclusion 2}

Comparing the margins of error, we note that The Method of trapezes and half-ellipses THE is more accurate and more precise than the following 3 methods:

- The method of rectangles on the left $\mathrm{R}_{\mathrm{n}}^{(1)}$,

- The method of rectangles on the right $\mathrm{R}_{\mathrm{n}}{ }^{(\mathrm{r})}$ and

- The method of trapezes $T_{n}$.

The approximation formula of The Method of trapezes and halfellipses given above can be considered as a special case of the following general formula:

$$
\begin{aligned}
& \int_{a}^{b} f(x) d x \approx T H E_{n}=\left(1-\frac{\gamma \pi}{4}\right) T_{n}+\left(\frac{\gamma \pi}{4}\right) M_{n} \\
& 0 \leq \gamma \leq \frac{4}{\pi} \&|e(\gamma, n)| \leq \frac{(b-a)^{3}}{12 n^{2}}\left(1-\frac{\gamma \pi}{8}\right) \sup _{[a, b]}\left|f^{\prime \prime}\right| \text { of course if } \mathrm{f} \text { is }
\end{aligned}
$$

$\mathrm{C}^{2}$ on $[\mathrm{a}, \mathrm{b}]$.
For $\gamma=0: \mathrm{THE}_{\mathrm{n}}=\mathrm{T}_{\mathrm{n}}$,

For $\gamma=\frac{4}{\pi}: \operatorname{THE}_{\mathrm{n}}=\mathrm{M}_{\mathrm{n}}$,

So, we can consider The method of trapezes and The method of rectangles (on the left, on the right and with midpoint) as special cases of the general method which is called The Method of trapezes and halfellipses THE.

\section{References}

1. Riemann B, Wilkins R (1998) On the Number of Prime Numbers less than a Given Quantity. Public university in Gottingen, Germany.

2. Julio CB (2012) Andrade Random Matrix Theory and £ Functions In Function Fi elds. School of Mathematics, University of Bristol, England.

3. Marek W (2010) Two arguments that the nontrivial zeros of the Ri emann zeta function are irrational. Cardinal Stefan Wyszynski University, Warszawa, Poland.

4. Borwein P, Choi S, Rooney B, Weirathmueller A (2010) The Riemann Hypothesis: A Resource for the Afficionado and Virtuoso Alike. Springer Berlin Heidelberg, Springer New York, pp: 1-533.

5. Murad AAA (2016) A Proof of the Ri emann Hypoth esis and Determination of the Relationship Between Non-Trivial Zeros of Zeta Functions and Prime Numbers. Mutah University, Jordan.

6. Garaev MZ, Yildmm CY (2017) On small distances between ordinat es of zeros of ((s) and ('( $s)$. Institute of Mathematics, Morelia, Mexico.

7. McPhedran RC (2018) Sum Rules for Functions of the Riemann Zeta Type. Univer sity of Sydney, Australia.

8. Vic Dannon H (2009) Riemann Zeta Function: The Ri emann Hypoth esis Origin, the Factorization Error, and the Count of the Primes. Gauge Institute Journal 5: 15-3. 